\title{
Ghost imaging using homodyne detection
}

\author{
M. Bache, ${ }^{*}$ E. Brambilla, A. Gatti, and L. A. Lugiato \\ INFM, Dipartimento di Fisica e Matematica, Università dell'Insubria, Via Valleggio 11, 22100 Como, Italy
}

(Received 20 February 2004; published 31 August 2004)

\begin{abstract}
We present a theoretical study of ghost imaging based on correlated beams arising from parametric downconversion, and which uses balanced homodyne detection to measure both the signal and idler fields. We analytically show that the signal-idler correlations contain the full amplitude and phase information about an object located in the signal path, both in the near-field and the far-field case. To this end we discuss how to optimize the optical setups in the two imaging paths, including the crucial point regarding how to engineer the phase of the idler local oscillator as to observe the desired orthogonal quadrature components of the image. As is well known, the near-field image resolution is inherently linked to the far-field bandwidth of the image, determined by the bandwidth of the source of the correlated beams. We show how to circumvent this limitation by using a spatial averaging technique which dramatically improves the imaging bandwidth of the far-field correlations as well as speeds up the convergence rate. The results are backed up by numerical simulations taking into account the finite size and duration of the pump pulse.
\end{abstract}

DOI: $10.1103 /$ PhysRevA.70.023823

PACS number(s): 42.50.Dv, 42.50.Ar, 42.30.Wb

\section{INTRODUCTION}

Ghost imaging relies on the spatial correlation between two beams created by, e.g., parametric down-conversion (PDC) [1-15]. Each of the correlated beams are sent through a distinct imaging system called the test arm and the reference arm. In the test arm an object is placed and the image of the object is then recreated from the spatial correlation function between the test and reference arm. A basic requisite of the ghost-imaging schemes is that by solely adjusting the reference arm setup and varying the reference pointlike detector position it should be possible to retrieve spatial information about the object, such as the object image (near field) and the object diffraction pattern (far field).

Traditionally the studies devoted to ghost imaging have considered PDC in the low-gain regime, where the conversion rate of the pump photons to a pair of entangled signalidler photons is low enough for the detector to resolve them one at a time. This leads to the so-called two-photon imaging schemes investigated by Klyshko [1,2]. The recreation of the object is then based on coincidence counts between the test and reference arm, and the working principles have been demonstrated experimentally [3-5]. Our group has focused on generalizing the governing PDC theory to the macroscopic, high-gain regime where the number of photons per mode is large [16-20]. Moreover, we generalized the theory behind the two-photon imaging schemes [1,2,6-9] to the high-gain regime [11-13] and also investigated the quantum properties of the signal-idler correlations in that case [17-20]. While coincidence detection is used for low gain, in the high-gain regime the object information is extracted from measuring pixel-by-pixel the signal-idler intensities and from this forming the correlations.

Here we consider a setup where the PDC beams are measured with balanced homodyne detection by overlapping

\footnotetext{
*Corresponding author: morten.bache@uninsubria.it
}

them with local oscillator (LO) fields. A similar scheme was studied previously by our group in the context of entanglement of the signal-idler beams from PDC [18], where it was used to show analytically that the entanglement is complete since it encompasses both the amplitudes and the phases of signal and idler. The initial motivation for using a homodyne scheme for ghost imaging came from the need to circumvent the problems related to information visibility in the macroscopic regime. Specifically, when intensity detection is performed a homogeneous background term is present in the measured correlation function $[11,12,20]$. This term, which can be rather large, does not contain any information about the object and lowers the image visibility. Instead, by using homodyne detection the signal-idler correlation becomes second order instead of fourth order, and hence this background term is absent. Another advantage of homodyne detection is that arbitrary quadrature components of the test and reference beams can be measured, which means that the homodyne detection scheme allows for both amplitude and phase measurements of the object. We will show detailed analytical calculations which demonstrate that this is indeed possible, both for the object image (near field) as well as its diffraction pattern (far field). It is possible to reconstruct even a pure phase object when a bucket detector is used in the test arm (in contrast to when intensity measurements are done, as it was shown for the coincidence counting case [14]).

We will present a technique that implements an average over the test detector position. This turns out to strongly improve the bandwidth of the reconstructed far-field pattern. Instead, in the traditional scheme where a fixed test detector position is used, the imaging bandwidth is limited to the far-field bandwidth of PDC. Additionally the method substantially speeds up the convergence rate of the far-field correlations. This implies that even complex diffraction patterns with high-frequency Fourier components can be reconstructed in a low number of pump pulses.

The suggested homodyne detection scheme is fairly complicated to implement experimentally, since it involves car- 
rying out two independent homodyne measurements of fields that are spatially and temporally multimode (with pulse durations on the order of ps). Even a single homodyne measurement of spatially multimode light has not previously been investigated in details to our knowledge (not even in the continuous-wave regime where the field is temporally single-mode). However, a dual homodyne measurement has been done in the case of spatially single-mode light [21,22]. In general, homodyne experiments of temporally multimode light have pointed out the importance of a proper overlap between the LO and the field [23,24], and alternative ways of producing the LO has been implemented [25] leading to a better overlap. An improper overlap leads to a degrading in, e.g., the squeezing as it effectively corresponds to a drop in the quantum efficiency [26]. In our case these problems are presumably less severe since the quantum efficiency does not play an important role. In fact, we will present numerical simulations that confirm that homodyne measurement of spatiotemporal multimode light can be done successfully, with the strongest technical restriction concerning the engineering of the phases of the local oscillators. It is worthwhile to mention that the homodyne measurement protocol (no background term in the correlations) in combination with the spatial averaging technique (increased spatial bandwidth) could open for the possibility of using an optical parametric oscillator below threshold for imaging. This would simplify a possible experimental implementation.

Recently a debate has been going on whether entanglement is necessary for extracting the information in ghost imaging [8,11-15,27]. In particular, our group has pointed out that the output beams obtained from impinging a thermal-like field on a beam splitter can be used in the same way as the entangled beams in a PDC setup [12], and this result was used in Ref. [27] for proposing a lensless x-raydiffraction scheme. However, using a thermal-like source makes it difficult to do homodyne measurements since there seems to be no fixed reference phase of the thermal-like field. Thus it seems that the homodyne technique does require a PDC setup to work, since this setup offers a fixed reference phase (that of the pump beam) which enables us to make averages over repeated pump shots.

The paper is structured as follows: In Sec. II the model for PDC is presented, and the optical setup of the imaging system is described in Sec. III. In Sec. IV we discuss the case where pointlike detectors are used in the test arm, while in Sec. V the bucket detector case is discussed. Both cases show analytical results in the stationary and plane-wave pump approximation concerning the retrieval of the image information of both the far and the near field, as well as numerical results that include the Gaussian profile of the pump. Besides confirming the analytical results these serve as examples for discussion and demonstration of the imaging performances of the system. In Sec. VI we draw the conclusions. Appendix A contains the explicit expressions of the PDC gain functions and shows a quadratic expansion of the gain phase, while Appendix B shows how to treat the temporal part of the analytical correlations.

\section{MODEL}

We use a general model describing the three-wave quantum interaction of PDC inside the nonlinear crystal $[19,20]$, which includes the effects of finite size and duration of the pump and the effects of spatiotemporal walk-off and groupvelocity dispersion. We consider a uniaxial $\chi^{(2)}$ nonlinear crystal cut for type-II phase matching. The injected beam at the frequency $\omega_{0}$ (pump beam) is sent into the crystal in the $z$ direction, and the pump photons can down-convert to sets of photons at the frequencies $\omega_{1}$ (signal photon, ordinarily polarized) and $\omega_{2}$ (idler photon, extraordinarily polarized).

The model consists of a set of operator equations describing the evolution of the quantum-mechanical boson operators for the signal and idler fields $a_{j}(\vec{x}, z, t)$ obeying at a given $z$ the commutator relations $\left[a_{i}(z, \vec{x}, t), a_{j}^{\dagger}\left(z, \vec{x}^{\prime}, t^{\prime}\right)\right]=\delta_{i j} \delta(\vec{x}$ $\left.-\vec{x}^{\prime}\right) \delta\left(t-t^{\prime}\right), i, j=1,2$, while all other combinations commute. The pump field is treated classically and taken as undepleted (the parametric approximation). Treating the operators in Fourier space $a_{j}(z, \vec{q}, \Omega)=\int(d \vec{x} / 2 \pi) \int(d t /$ $\sqrt{2 \pi}) a_{j}(z, \vec{x}, t) e^{-i \vec{q} \cdot \vec{x}+i \Omega t}, j=1,2$, the signal equation of evolution is then

$$
\begin{aligned}
\mathcal{L}_{1}(z, \vec{q}, \Omega) a_{1}(z, \vec{q}, \Omega)= & \sigma e^{-i \Delta_{0} z} \int \frac{d \vec{q}^{\prime}}{2 \pi} \int \frac{d \Omega^{\prime}}{\sqrt{2 \pi}} A_{0}\left(z, \vec{q}-\vec{q}^{\prime}, \Omega\right. \\
& \left.-\Omega^{\prime}\right) a_{2}^{\dagger}\left(z,-\vec{q}^{\prime},-\Omega^{\prime}\right),
\end{aligned}
$$

while the idler equation can be found by exchanging subscripts $1 \leftrightarrow 2$. $\sigma$ is the strength of the nonlinearity, while $\Delta_{0} \equiv k_{1}+k_{2}-k_{0}$ denotes the collinear phase mismatch between the three waves along the $z$ axis. The linear propagation operator is defined as $\mathcal{L}_{j}(z, \vec{q}, \Omega) \equiv \partial / \partial z-i \delta_{j}(\vec{q}, \Omega)$, where

$$
\delta_{j}(\vec{q}, \Omega)=k_{j}^{\prime} \Omega+\frac{1}{2} k_{j}^{\prime \prime} \Omega^{2}+\rho_{j} q_{x}-\frac{1}{2 k_{j}}|\vec{q}|^{2} .
$$

$k_{j}=n_{j} \omega_{j} / c$ is the wave number of field $j$ inside the crystal, and $k_{j}^{\prime}=\partial k_{j} /\left.\partial \omega\right|_{\omega=\omega_{j}}$ describes temporal walk-off while $k_{j}^{\prime \prime}$ $=\partial^{2} k_{j} /\left.\partial \omega^{2}\right|_{\omega=\omega_{j}}$ describes group velocity dispersion. $\rho_{j}$ $=\partial k /\left.\partial q_{x}\right|_{\vec{q}=0}$ is the walk-off angle. The classical pump is in the numerics taken as being Gaussian in both time and space $A_{0}(z=0, \vec{x}, t) \propto A_{p} e^{-|\vec{x}|^{2} / w_{0}^{2}-t^{2} / \tau_{0}^{2}}$, where $w_{0}$ and $\tau_{0}$ are the pump waist and duration time, while $A_{p}$ is the pump amplitude. For additional details about the origin of the model see, e.g., Ref. [20].

In the stationary and plane-wave pump approximation (SPWPA) the pump is taken as translationally invariant as well as continuous wave. Under this condition Eqs. (1) can be solved analytically. Thus the unitary input-output transformations relating the field operators at the output face of the crystal $a_{j}^{\text {out }}(\vec{q}, \Omega) \equiv a_{j}\left(z=l_{c}, \vec{q}, \Omega\right)$ to those at the input face $a_{j}^{\text {in }}(\vec{q}, \Omega) \equiv a_{j}(z=0, \vec{q}, \Omega)$ then take the following form:

$$
\begin{aligned}
a_{i}^{\text {out }}(\vec{q}, \Omega)= & U_{i}(\vec{q}, \Omega) a_{i}^{\text {in }}(\vec{q}, \Omega)+V_{i}(\vec{q}, \Omega) a_{j}^{\text {in } \dagger}(-\vec{q},-\Omega), \\
& i \neq j=1,2 .
\end{aligned}
$$

The gain functions $U_{j}$ and $V_{j}$ are given in Appendix A.

\section{SYSTEM SETUP}

Ghost imaging is characterized by its two-arm configuration, with an unknown object placed in the test arm. The 
(a)

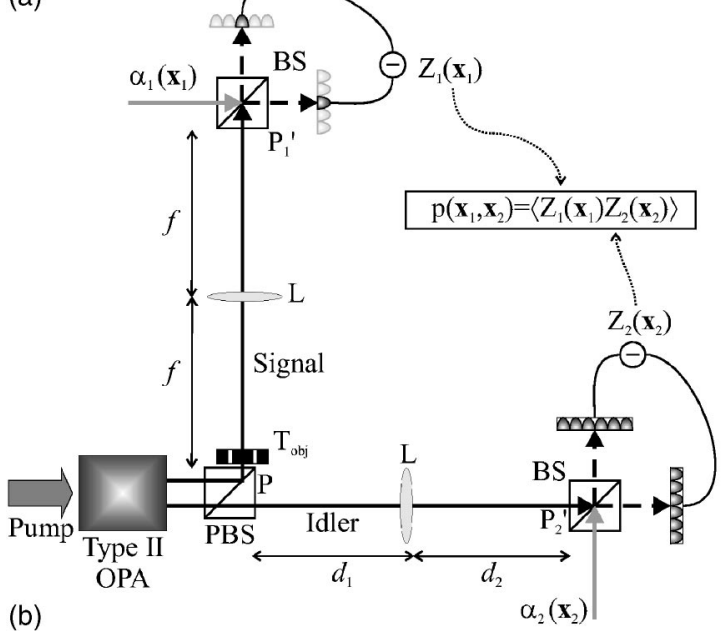

(b)

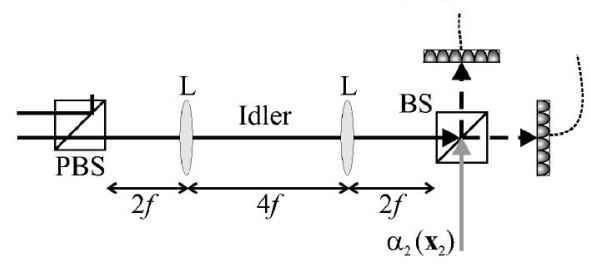

(c)

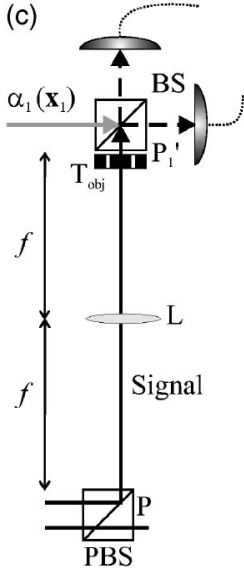

FIG. 1. The system setup. Sketch (a) shows the general setup where a pointlike detector (or a pixel array) is used in the test (signal) arm. Sketch (b) shows the case of a telescope setup in the reference (idler) arm. Sketch (c) shows the test arm setup when bucket detectors are used for the measurements there. See the text for more details. $L$, lens of focal length $f$; PBS, polarized beam splitter; BS, beam splitter; $P$, crystal plane; $P_{1}^{\prime}$, signal measurement plane; $P_{2}^{\prime}$, idler measurement plane. information about the object is retrieved from the cross correlations of the fields recorded in the test and reference arms as a function of the reference arm pixel position; see Fig. 1. By simply changing the optical setup in the reference arm, information about both the object image (near field) and the object diffraction pattern (far field, i.e., the Fourier transform) can be obtained. There are two main motivations for using such an imaging configuration. First, this configuration makes it possible to do coherent imaging even if each of the two fields are spatially incoherent (and thus recording the far-field spatial distribution in the test arm does not give any information about the object diffraction pattern). Second, the configuration allows for a simple detection protocol in the test arm even without measuring spatial information, and still the spatial information can be retrieved from the correlations. Thus a simple bucket detector setup can be used that collects all photons, or alternatively using a single pixel detector (pointlike detector). This is advantageous when the object is located in a environment that is hard to access making it difficult to place an array of detectors after the object, or if the stability of the test arm is an issue making a simple setup crucial.

We now show how to evaluate the signal and idler fields as they propagate through the two-arm configuration, and how to evaluate the resulting correlations between them. The type-II phase matching conditions of the crystal ensure that the signal and idler have orthogonal polarizations and therefore at the exit of the crystal they can be separated by a polarizing beam splitter (PBS). We will neglect the distance between the crystal and the PBS. The fields $c_{j}(\vec{x}, t)$ at the measurement planes $P_{j}^{\prime}$ are connected to the output fields at the crystal exit plane $P$ by a Fresnel transformation formally written as

$$
c_{j}\left(\vec{x}_{j}, t\right)=\int d \vec{x}^{\prime} h_{j}\left(\vec{x}_{j}, \vec{x}^{\prime}\right) a_{j}^{\text {out }}\left(\vec{x}^{\prime}, t\right)+L_{j}\left(\vec{x}_{j}\right),
$$

where $h_{j}\left(\vec{x}_{j}, \vec{x}^{\prime}\right)$ are kernels related to the optical path from $P$ to $P_{j}^{\prime} . L_{j}\left(\vec{x}_{j}\right)$ accounts for losses in the imaging system that are linearly proportional to vacuum field operators and therefore uncorrelated to the PDC fields.

The object is described by a transmission function $T_{\mathrm{obj}}(\vec{x})$, and is set in the signal arm. Besides the object the signal arm is set in the so-called $f-f$ imaging scheme, consisting of a lens $L$ with focal length $f$ located at a distance $f$ from both the crystal exit and from the detection plane $P_{1}^{\prime}$. It will emerge from our analysis that when a pointlike detector is used the object must be placed at $P$, as shown in Fig. 1(a). Conversely, when bucket detectors are used the object must be placed at $P_{1}^{\prime}$, as shown in Fig. 1(c). Consequently, the signal Fresnel kernels are

$$
\begin{aligned}
& h_{1}\left(\vec{x}_{1}, \vec{x}^{\prime}\right)=\left(i \lambda_{1} f\right)^{-1} e^{-i\left(2 \pi / \lambda_{1} f\right) \vec{x}_{1} \cdot \vec{x}^{\prime}} T_{\mathrm{obj}}\left(\vec{x}^{\prime}\right), \\
& h_{1}^{\mathrm{b}}\left(\vec{x}_{1}, \vec{x}^{\prime}\right)=\left(i \lambda_{1} f\right)^{-1} e^{-i\left(2 \pi / \lambda_{1} f\right) \vec{x}_{1} \cdot \vec{x}^{\prime}} T_{\mathrm{obj}}\left(\vec{x}_{1}\right),
\end{aligned}
$$

for the pointlike and the bucket detector case, respectively, and the superscript " $b$ " denotes bucket. $\lambda_{1}$ is the wavelength of the signal field. Note that the lens in the signal arm could actually be removed and substituted by a propagation over a distance long enough to be in the Frauenhofer regime. However, the phase of the signal field could be difficult to control in that case.

We will fix the setup of the signal arm once and for all. We may then adjust the idler arm to retrieve the desired kind of information through the signal-idler correlations. In one setup the idler lens system also consists of a lens $L$ of focal length $f$, placed a distance $d_{1}$ from $P$ and $d_{2}$ from $P_{2}^{\prime}$ [see 
Fig. 1(a)]. If $d_{1}$ and $d_{2}$ are identical and both equal to the lens focal length, $d_{1}=d_{2}=f$, we have an $f-f$ setup as also used in the signal arm. As we will show, with this setup the correlations in the pointlike detector case give information about the object far field, while in the bucket detector case they give information about the object near field.

In another setup the idler arm consists of a telescopic system made of two identical lenses $L$ swith focal length $f$, each placed a distance $2 f$ from $P$ and $P_{2}^{\prime}$, respectively, and with a distance $4 f$ between them, see Fig. 1(b). This setup images point by point the near field at the plane $P$ to the plane $P_{2}^{\prime}$. As we will show, with this configuration the correlations provide information about the object near field for the pointlike detector case, while the object far field can be retrieved in the bucket detector case.

For these two chosen setups in the reference arm, the field at the reference detection plane $P_{2}^{\prime}$ is then given by the Fresnel transformation (4) with the kernels

$$
\begin{gathered}
h_{2, f}\left(\vec{x}, \vec{x}^{\prime}\right)=\left(i \lambda_{2} f\right)^{-1} e^{-i\left(2 \pi / \lambda_{2} f\right) \vec{x} \cdot \vec{x}^{\prime}}, \\
h_{2, T}\left(\vec{x}, \vec{x}^{\prime}\right)=\delta\left(\vec{x}-\vec{x}^{\prime}\right),
\end{gathered}
$$

for the $f-f$ setup and the telescope setup, respectively. Note that a $2 f-2 f$ setup - obtained by setting $d_{1}=d_{2}=2 f$ in Fig. 1(a) —would also provide an image plane at $P_{2}^{\prime}$. We choose to work with the telescope setup with respect to such a $2 f-2 f$ setup in order to avoid the introduction of a wave-front curvature which could be a nuisance in a phase sensitive measurement.

The fields at $P_{j}^{\prime}$ are measured using balanced homodyne detection schemes, so $c_{j}\left(\vec{x}_{j}, t\right)$ is mixed with an LO $\alpha_{j}\left(\vec{x}_{j}, t\right)$ on a 50/50 beam splitter, and after the beam splitter the two fields are $c_{j, \pm}(\vec{x}, t)=\left[c_{j}(\vec{x}, t) \pm \alpha_{j}(\vec{x}, t)\right] / \sqrt{2}$. The LO is treated as a classical coherent field and therefore by measuring the field intensities at the photodetectors and subtracting them we obtain

$$
\begin{aligned}
Z_{j}(\vec{x}, t) \equiv & c_{j,+}^{\dagger}(\vec{x}, t) c_{j,+}(\vec{x}, t)-c_{j,-}^{\dagger}(\vec{x}, t) c_{j,-}(\vec{x}, t)=\left|\alpha_{j}(\vec{x}, t)\right| \\
& \times\left[c_{j}(\vec{x}, t) e^{-i \phi_{j}^{\mathrm{LO}}(\vec{x}, t)}+c_{j}^{\dagger}(\vec{x}, t) e^{i \phi_{j}^{\mathrm{L}(}(\vec{x}, t)}\right] .
\end{aligned}
$$

Here we have the well-known result that by properly adjusting the local oscillator phase $\phi_{j}^{\mathrm{LO}}(\vec{x}, t)$ one can measure a particular quadrature component of the field.

We now consider the correlation between two particular quadrature components of the signal and idler fields, $Z_{1}$ and $Z_{2}$, integrated over a finite detection time $T_{d}$,

$$
\begin{aligned}
p\left(\vec{x}_{1}, \vec{x}_{2}\right)= & \int_{T_{d}} d t_{1} \int_{T_{d}} d t_{2}\left\langle Z_{1}\left(\vec{x}_{1}, t_{1}\right) Z_{2}\left(\vec{x}_{2}, t_{2}\right)\right\rangle \\
= & \int_{T_{d}} d t_{1} \int_{T_{d}} d t_{2} \alpha_{1}^{*}\left(\vec{x}_{1}, t_{1}\right) \alpha_{2}^{*}\left(\vec{x}_{2}, t_{2}\right) \\
& \times\left\langle c_{1}\left(\vec{x}_{1}, t_{1}\right) c_{2}\left(\vec{x}_{2}, t_{2}\right)\right\rangle+\text { c.c. }
\end{aligned}
$$

The last line follows from adopting the parametric approximation as well as from the fact that the signal and idler are in the vacuum state at the crystal entrance, since under these assumptions $\left\langle c_{1}^{\dagger}\left(\vec{x}_{1}, t_{1}\right) c_{2}\left(\vec{x}_{2}, t_{2}\right)\right\rangle=0$. In most cases $\left.\left.T_{d}\right\rangle\right\rangle \tau_{\text {coh }}$ so the detector is too slow to follow the temporal dynamics of the system.

The analytical calculations are based on the SPWPA, under which we may evaluate the correlation (8b) using the input-output relations (3). In the degenerate case the signal and idler have identical wavelengths $\lambda_{1}=\lambda_{2} \equiv \lambda$ and hence also vacuum wave numbers $k_{1, \mathrm{v}}=k_{2, \mathrm{v}} \equiv k_{\mathrm{v}}=2 \pi / \lambda$. In order to evaluate the correlation (8b) we note that Eq. (4) provides the link between the detection plane and the exit of the crystal, and there the correlations are the near field signal-idler correlation which can be found from Eq. (A3b) as

$$
\begin{aligned}
& \left\langle a_{1}^{\text {out }}\left(\vec{x}_{1}, t_{1}\right) a_{2}^{\text {out }}\left(\vec{x}_{2}, t_{2}\right)\right\rangle \\
& =\int \frac{d \Omega}{2 \pi} e^{-i \Omega\left(t_{1}-t_{2}\right)} \int \frac{d \vec{q}}{(2 \pi)^{2}} e^{i \vec{q}\left(\vec{x}_{1}-\vec{x}_{2}\right)} \gamma(\vec{q}, \Omega),
\end{aligned}
$$

where we have introduced the gain function

$$
\gamma(\vec{q}, \Omega)=|\gamma(\vec{q}, \Omega)| e^{i \phi_{\gamma}(\vec{q}, \Omega)} \equiv U_{1}(\vec{q}, \Omega) V_{2}(-\vec{q},-\Omega) .
$$

Appendix A shows an approximate quadratic expansion of the gain phase that will become useful in the following.

We now use Eqs. (4) and (9) to obtain a more useful notation for the signal-idler correlation at the detectors,

$$
\begin{aligned}
&\left\langle c_{1}\left(\vec{x}_{1}, t_{1}\right) c_{2}\left(\vec{x}_{2}, t_{2}\right)\right\rangle= \int \frac{d \Omega}{2 \pi} e^{-i \Omega\left(t_{1}-t_{2}\right)} \\
& \times \int d \vec{q} h_{1}\left(\vec{x}_{1},-\vec{q}\right) h_{2}\left(\vec{x}_{2}, \vec{q}\right) \gamma(\vec{q}, \Omega), \\
& h_{j}(\vec{x}, \vec{q}) \equiv \int \frac{d \vec{x}^{\prime}}{2 \pi} e^{-i \vec{q} \cdot \vec{x}^{\prime}} h_{j}\left(\vec{x}, \vec{x}^{\prime}\right) .
\end{aligned}
$$

By using Eq. (11a) we may evaluate the correlation (8b) for $\left.\left.T_{d}\right\rangle\right\rangle \tau_{\text {coh }}$. The details of this calculation are put in Appendix $\mathrm{B}$, and basically there are two limits to consider. First, for a pulsed LO which has a duration much smaller than $\tau_{\text {coh }}$ the result is given by Eq. (B2). Second, for a continuous-wave LO which has a duration much larger than $\tau_{\text {coh }}$ the result is given by Eq. (B3). Since we did not find substantial differences between the two cases, we decided to use Eq. (B3) in the rest of the paper for the analytical part. It states that in the limit of a continuous-wave $L O$ the signal-idler quadrature correlation is

$$
\begin{aligned}
& \begin{aligned}
p\left(\vec{x}_{1}, \vec{x}_{2}\right)= & \frac{T_{d}}{2 \pi} \alpha_{1}^{*}\left(\vec{x}_{1}\right) \alpha_{2}^{*}\left(\vec{x}_{2}\right) \int d \vec{q} h_{1}\left(\vec{x}_{1},-\vec{q}\right) h_{2}\left(\vec{x}_{2}, \vec{q}\right) \gamma(\vec{q}, 0) \\
& + \text { c.c., }
\end{aligned} \\
& \text { where } \alpha_{j}(\vec{x}) \equiv \alpha_{j}(\vec{x}, t=0) .
\end{aligned}
$$

\section{POINTLIKE DETECTORS IN THE TEST ARM}

Using a pointlike detector setup in the test arm is the typical configuration used in ghost imaging. Therefore the results of this section are similar to those obtained previously 
using an intensity detection scheme, both by our group in the high-gain regime [11-13], as well as those obtained by others in the low-gain (two-photon) regime [1-3,5-10,15]. The differences between the homodyne detection case, which we will now show, and the papers mentioned above are that we here do not have problems with visibility and that we can access arbitrary quadrature components of the object distribution function.

We use here the setup of Fig. 1(a), so the test arm we keep fixed in the $f$ - $f$ setup with sthe object at the crystal exit. The idler arm is then set in either the $f$ - $f$ setup [with $d_{1}=d_{2}=f$ in Fig. 1(a)] or the telescope setup [Fig. 1(b)]. The information is extracted by fixing the signal detector position $\vec{x}_{1}$ and scanning the idler detector position $\vec{x}_{2}$. This gives a simple, fixed setup of the test arm. Alternatively, we may also scan the signal detector position and thereby we can achieve an improved imaging bandwidth and convergence rate for the diffraction pattern (the spatial average technique).

\section{A. Analytical results}

In order to evaluate analytically the quadrature correlation (12) for pointlike detectors in the test arm the kernel (5a) must be used in the form of Eq. (11b). Then, as we shall now show, using the kernels (6a) and (6b) gives either the object far field or the object near field.

\section{Retrieval of the object far field}

The object far field can be retrieved from the correlations by using the $f-f$ scheme in the reference arm, implying that we must use the kernel (6a) in the form (11b). By using these in Eq. (12) the signal-idler quadrature correlation for retrieving the object far field is

$$
\begin{aligned}
& p_{f}\left(\vec{x}_{1}, \vec{x}_{2}\right)= \frac{2 T_{d}}{(\lambda f)^{2}}\left|\gamma\left(-\vec{x}_{2} k_{\mathrm{v}} / f, 0\right) \alpha_{1}\left(\vec{x}_{1}\right) \alpha_{2}\left(\vec{x}_{2}\right)\right| \operatorname{Re}\left(\widetilde { T } _ { \mathrm { obj } } \left[\left(\vec{x}_{1}\right.\right.\right. \\
&\left.\left.\left.+\vec{x}_{2}\right) k_{\mathrm{v}} / f\right] e^{i \Phi_{f}\left(\vec{x}_{1}, \vec{x}_{2}\right)}\right), \\
& \Phi_{f}\left(\vec{x}_{1}, \vec{x}_{2}\right) \equiv \phi_{\gamma}\left(-\vec{x}_{2} k_{\mathrm{v}} / f, 0\right)-\phi_{2, f}^{\mathrm{LO}}\left(\vec{x}_{2}\right)-\phi_{1}^{\mathrm{LO}}\left(\vec{x}_{1}\right)+\pi,
\end{aligned}
$$

where $\widetilde{T}_{\text {obj }}(\vec{q})$ is the Fourier transform of $T_{\text {obj }}(\vec{x})$.

We now discuss how to optimize the system for obtaining the far-field image information. First of all we notice that we need to keep the spatial dependence of the LO moduli as constant as possible, effectively setting a limit on their spatial waists. However, since the effect of the LO moduli is a simple multiplication on the correlation it is easy to do a post measurement correction of the correlations. This means that matching the spatial overlaps of the LO moduli and the PDC fields should be straightforward, while the phases of the LO's are more crucial, as discussed below. The same comments turn out to hold for the other setups discussed in the paper.

Second of all, when $\vec{x}_{2}$ is scanned the gain modulus comes into play. We choose a noncollinear phase-matching condition (see Sec. IV B in which $\left|\gamma\left(\vec{q}=-\vec{x}_{2} k_{\mathrm{v}} / f, 0\right)\right|$ is a plateau shaped function centered on $\vec{q}=-\vec{q}_{C}$, with [20]

$$
\vec{q}_{C}=\frac{1}{2} \vec{\rho}_{2} l_{c} q_{0}^{2}=\vec{\rho}_{2}\left(1 / k_{1}+1 / k_{2}\right)^{-1}
$$

Additionally, it is roughly constant over a finite region determined by the spatial bandwidth $q_{0}$ defined by Eq. (A4a). This sets a limit to the imaging bandwidth since the higher-order spatial frequency components are cut off by the gain. That the source of the correlated beams acts as a low-pass spatial frequency filter is well known, but in Sec. IV B 3 we will show how to circumvent this limitation using the spatial average technique.

Finally, the most crucial point is to control the LO phases to observe the desired quadrature components of the object far field. It is seen that the real part of the object far field is obtained if we can make $\Phi_{f}\left(\vec{x}_{1}, \vec{x}_{2}\right)=0$, while if we can make $\Phi_{f}\left(\vec{x}_{1}, \vec{x}_{2}\right)=-\pi / 2$ the imaginary part of the object far field is obtained. This can be achieved by engineering $\phi_{2, f}^{\mathrm{LO}}\left(\vec{x}_{2}\right)$ to cancel the gain phase dependence on $\vec{x}_{2}$ that appears in Eq. (13b). The idler LO phase is in general composed as follows:

$$
\phi_{2, f}^{\mathrm{LO}}\left(\vec{x}_{2}\right)=\psi_{2, f}^{\mathrm{LO}}+\vec{q}_{2, f}^{\mathrm{LO}} \cdot \vec{x}_{2},
$$

where $\psi_{2, f}^{\mathrm{LO}}$ is a controllable reference phase and the wave number $\vec{q}_{2, f}^{\mathrm{LO}}$ may be applied to the LO by using a grating. As a good approximation $\phi_{\gamma}\left(-\vec{x}_{2} k_{v} / f, 0\right)$ is constant because it is a slow function over a region determined by $q_{0}$ (we should consequently evaluate the gain at its maximum, i.e., at the gain center $-\vec{x}_{2} k_{\mathrm{v}} / f=-\vec{q}_{C}$ ). We may therefore take $\phi_{2, f}^{\mathrm{LO}}\left(\vec{x}_{2}\right)$ constant $\left(\vec{q}_{2, f}^{\mathrm{LO}}=0\right)$, so to obtain the quadrature corresponding to the real part we should choose the reference phase

$$
\psi_{2, f}^{\mathrm{LO}}=\phi_{\gamma}\left(-\vec{q}_{C}, 0\right)-\phi_{1}^{\mathrm{LO}}\left(\vec{x}_{1}\right)+\pi
$$

The quadrature component corresponding to the imaginary part of the object far field may consequently be observed by adding $\pi / 2$ to this value.

We can improve this result by using that, as shown in Appendix A, the gain phase can be approximated with a quadratic expansion in $\vec{q}$, i.e., $\quad \phi_{\gamma}(\vec{q}, 0) \simeq \phi_{\gamma}^{(0)}+\vec{\phi}_{\gamma, q}^{(1)} \cdot \vec{q}$ $+\phi_{\gamma, q}^{(2)}|\vec{q}|^{2}$ [see Eq. (A8a)]. The linear term $\vec{\phi}_{\gamma, q}^{(1)}$ can be compensated for by making the idler LO a tilted wave, i.e., choosing

$$
\vec{q}_{2, f}^{\mathrm{LO}}=-\vec{\phi}_{\gamma}^{(1)} k_{\mathrm{v}} / f=\rho_{2} l_{c} \Psi{ }_{\gamma} / f \vec{e}_{x} .
$$

The quadratic term $\phi_{\gamma, q}^{(2)}$ may be cancelled by shifting the focusing plane of the idler $f$ - $f$ system $\Delta z$ away from the crystal plane $P$ [20], since this gives (in Fourier space) a Fresnel transformation contribution of $\exp \left(-i \Delta z|\vec{q}|^{2} / 2 k_{\mathrm{v}}\right)$ on the gain function in Eq. (9). By setting $\Delta z=\phi_{\gamma}^{(2)} 2 k_{\mathrm{v}}$ the quadratic phase term is exactly cancelled. So we must image a plane inside the crystal since this choice from Eq. (A8d) implies

$$
\Delta z=-\left(1 / n_{1}+1 / n_{2}\right) \Psi_{\gamma} l_{c},
$$

with $\Psi_{\gamma}$ defined in Eq. (A7). Now all we need to do is to find the overall reference phase. To observe the quadrature component corresponding to the real part of the object far field we should choose 


$$
\psi_{2, f}^{\mathrm{LO}}=\phi_{\gamma}^{(0)}-\phi_{1}^{\mathrm{LO}}\left(\vec{x}_{1}\right)+\pi,
$$

with $\phi_{\gamma}^{(0)}$ given by Eq. (A8b). This result, the tilted wave LO (17) and the shift of the focusing plane (18) represent the optimized scheme for accessing the object far field. The reference phase without optimizing is given by Eq. (16).

\section{Retrieval of object near field}

The object near field can be observed by using the telescope setup in the reference arm, thus using the idler kernel (6b) in the form (11b). Then Eq. (12) becomes

$$
\begin{aligned}
p_{T}\left(\vec{x}_{1}, \vec{x}_{2}\right)= & \frac{T_{d} \alpha_{1}^{*}\left(\vec{x}_{1}\right) \alpha_{2}^{*}\left(\vec{x}_{2}\right)}{2 \pi i \lambda f} e^{-i \vec{x}_{1} \cdot \vec{x}_{2} k_{v} / f} \\
& \times \int \frac{d \vec{q}}{2 \pi} e^{-i \vec{q} \cdot \vec{x}_{2}} \widetilde{T}_{\mathrm{obj}}(\vec{q}) \gamma\left(\vec{x}_{1} k_{v} / f-\vec{q}, 0\right)+\text { c.c. }
\end{aligned}
$$

The correlation is therefore an integral over the gain function and the object far field. Since the gain modulus is a roughly plateau-shaped function in a region determined by $q_{0}$ that acts as a cutoff of the higher values of $\vec{q}$, and the gain phase dependence is slow within this region, the gain can as a good approximation be pulled out of the integration as a constant evaluated at the center of maximum gain $\vec{q}=-\vec{q}_{C}$. The remaining integral is merely the inverse Fourier transform of the object far field, i.e., simply the object near field. Hence the quadrature correlation for retrieving the object near field is

$$
\begin{gathered}
p_{T}\left(\vec{x}_{1}, \vec{x}_{2}\right) \simeq \frac{T_{d}\left|\gamma\left(-\vec{q}_{C}, 0\right) \alpha_{1}\left(\vec{x}_{1}\right) \alpha_{2}\left(\vec{x}_{2}\right)\right|}{\pi \lambda f} \\
\times \operatorname{Re}\left[T_{\mathrm{obj}}\left(\vec{x}_{2}\right) e^{i \Phi_{T}\left(\vec{x}_{1}, \vec{x}_{2}\right)}\right] \\
\Phi_{T}\left(\vec{x}_{1}, \vec{x}_{2}\right) \equiv-\vec{x}_{2} \cdot \vec{x}_{1} k_{\mathrm{v}} / f+\phi_{\gamma}\left(-\vec{q}_{C}, 0\right)-\phi_{1}^{\mathrm{LO}}\left(\vec{x}_{1}\right)-\phi_{2, T}^{\mathrm{LO}}\left(\vec{x}_{2}\right) \\
-\pi / 2 .
\end{gathered}
$$

We stress that it is only an approximate result that neglected a cutoff of the high-frequency components of the object Fourier transform. Thus image information that relies on large spatial frequency components will not be reproduced by the correlations. This is the well-known result that the near-field resolution is limited by the far-field gain bandwidth.

The first step in engineering $\Phi_{T}\left(\vec{x}_{1}, \vec{x}_{2}\right)$ as to observe a given quadrature component consists in taking care of the linear contribution in $\vec{x}_{2}$. In the spirit of Eq. (15), we take $\phi_{2, T}^{\mathrm{LO}}\left(\vec{x}_{2}\right)=\psi_{2, T}^{\mathrm{LO}}+\vec{q}_{\mathrm{LO}}^{2, T} \cdot \vec{x}_{2}$ and choose

$$
\vec{q}_{2, T}^{\mathrm{LO}}=-\vec{x}_{1} k_{v} / f
$$

Thus making the idler LO a tilted wave is crucial to observe the near-field distribution. Moreover, in order to observe the real part the overall constant reference phase must be chosen to

$$
\psi_{2, T}^{\mathrm{LO}}=\phi_{\gamma}\left(-\vec{q}_{C}, 0\right)-\phi_{1}^{\mathrm{LO}}\left(\vec{x}_{1}\right)-\pi / 2 .
$$

We may reduce the approximation made in going from Eq. (20) to Eq. (21a) by compensating the quadratic term of the gain phase by taking the imaging plane inside the crystal by the amount(18), in the same way as discussed in the previous section. Thus when the setup is optimized the idler reference phase should instead be

$$
\psi_{2, T}^{\mathrm{LO}}=\phi_{\gamma}^{(0)}-\phi_{1}^{\mathrm{LO}}\left(\vec{x}_{1}\right)+\vec{x}_{1} \cdot \vec{\phi}_{\gamma}^{(1)} k_{v} / f-\pi / 2,
$$

with $\phi_{\gamma}^{(0)}$ and $\vec{\phi}_{\gamma}^{(1)}$ given in Eq. (A8).

\section{B. Numerical results}

In this subsection we show specific examples from numerical simulations that support the analytical results obtained in the pointlike detector case. Additionally we introduce the spatial average technique.

\section{General introduction}

The numerical simulations presented in this paper take into account the Gaussian profile as well as the pulsed character of the pump. They consisted of solving the Eqs. (1) using the Wigner representation to write the stochastic equations. At each pump shot a stochastic field was generated at the crystal input with Gaussian statistics corresponding to the vacuum state, and quantum expectation values corresponding to symmetrical ordering were obtained by averaging over pump shots. See Ref. [20] for more details. The system we have chosen to investigate is the setup of a current experiment performed in Como [28,29]. Thus we consider a BBO crystal cut for type-II phase matching. The pump pulse is at $\lambda_{0}=352 \mathrm{~nm}$ and has a duration $\tau_{0}=1.5 \mathrm{ps}$. The crystal length is $l_{c}=4 \mathrm{~nm}$ and the dimensionless gain parameter $\sigma_{p} l_{c}=4.0$. Together with a pump waist of $w_{0}=660 \mu \mathrm{m}$ it corresponds roughly to a pump pulse energy of $200 \mu \mathrm{J}$. The crystal is collinearly phase matched at an angle $\theta=49.05^{\circ}$ of the pump propagation direction with respect to the optical $z$ axis [30]. We used $\theta=48.2^{\circ}$ which gives $\Delta_{0}<0$ and a gain that is plateau shaped over a large region centered on $-\vec{q}_{C}$ as given by Eq. (14). With this setup the typical time and length scales (A5) are $x_{\text {coh }}=17 \mu \mathrm{m}$ and $\tau_{\text {coh }}=0.96$ ps.

The correlations typically converged after a couple of thousands repeated pump shots when no spatial information was recorded in the test beam (i.e., using either bucket or pointlike detectors there). For this reason it is very time consuming to do a full $(3+1) \mathrm{D}$ simulation ( $x y t$ propagated along $z)$. Instead we chose to model a $(2+1) \mathrm{D}$ system: a single transverse dimension $x$ along the walk-off direction and the temporal dimension $t$ with propagation along the crystal direction $z$. We will also show simulations where in addition to averaging over shots, a spatial average over $\vec{x}_{1}$ is also performed. In the $(2+1) \mathrm{D}$ simulations this reduces the number of shots needed by an order of magnitude, while in (3 $+1) \mathrm{D}$ it is possible to average over both transverse directions and a diffraction image can be observed even with only a few shots. Thus this method allows us to calculate the correlations even from a full $(2+1) \mathrm{D}$ simulation.

The integration along the crystal direction was done in $N_{z}=200$ steps. In the $(2+1) \mathrm{D}$ simulations the $x t$ grid was chosen to $N_{x}=512$ and $N_{t}=32$, while for the $(3+1) \mathrm{D}$ simulations we chose $N_{x}=N_{y}=256$ and $N_{t}=32$. $(\vec{x}, t)$ were scaled 
to $x_{\text {coh }}$ and $\tau_{\text {coh }}$, respectively, as given by Eq. (A5), while $z$ was scaled to the crystal length $l_{c}$.

We chose to investigate the quantitative behavior of the imaging system with a transmitting double slit as the object, defined in one transverse dimension as

$$
T_{\mathrm{obj}}(x)=\left\{\begin{array}{l}
0, \quad\left|x-\delta_{x}\right|>\frac{a+d}{2}, \quad\left|x-\delta_{x}\right|<\frac{d-a}{2} \\
1, \quad \frac{d-a}{2} \leqslant\left|x-\delta_{x}\right| \leqslant \frac{a+d}{2},
\end{array}\right.
$$

where $d$ is the distance between the slit centers, $a$ is the slit width, and $\delta_{x}$ is the object shift from origin. This object only introduces amplitude modulations and does not alter phase information. We take $\delta_{x} \neq 0$ in the numerics to compensate for walk-off effects, so we must choose it properly in order for each slit to see the same gain. The analytical Fourier transform is

$$
\widetilde{T}_{\mathrm{obj}}(q)=a \sqrt{2 / \pi} e^{-i \delta_{x} q} \cos (q d / 2) \operatorname{sinc}(q a / 2),
$$

where $\operatorname{sinc}(x)=\sin (x) / x$. The shift $\delta_{x}$ alone introduces a nonzero imaginary part, but even without such a shift the double slit diffraction pattern contains phase information (it is both positive and negative). Additionally we will present results using a pure phase double slit [10]

$$
T_{\text {obj }}(x)=\left\{\begin{array}{l}
1, \quad\left|x-\delta_{x}\right|>\frac{a+d}{2}, \quad\left|x-\delta_{x}\right|<\frac{d-a}{2} \\
-1, \quad \frac{d-a}{2} \leqslant\left|x-\delta_{x}\right| \leqslant \frac{a+d}{2} .
\end{array}\right.
$$

This object does not alter amplitude information.

In homodyne detection the LO is typically taken from the same source that created the pump. One would first generate the pump through second-harmonic generation (SHG), and before the laser light at the fundamental frequency enters the SHG crystal some of the power is taken out using a beam splitter, and this field is then used for the LO's. The second harmonic at the exit of the SHG process is then the pump for the PDC process. Therefore the LO's have more or less the same energy, shape and duration as the pump pulse entering the PDC setup. Through numerical simulations with Gaussian LO's we confirmed the results indicated in the analytical sections, namely that the effect of a Gaussian shaped LO appears trivially as a multiplication [see Eqs. (13a) and (21)]. Since broader LO's can easily be achieved experimentally using a beam expander, we decided to keep the LO's as plane waves in space. Also this allows to base the interpretation of the results on the physics behind the PDC and keeping in mind that the LO's will only change the result with a multiplication of a Gaussian function. The temporal duration was chosen identical to the pump (1.5 ps), which ensures a good overlap between the pulses [26].

\section{Averaging over shots}

In this section we present numerical results from using pointlike detectors in the test arm and averaging over re-
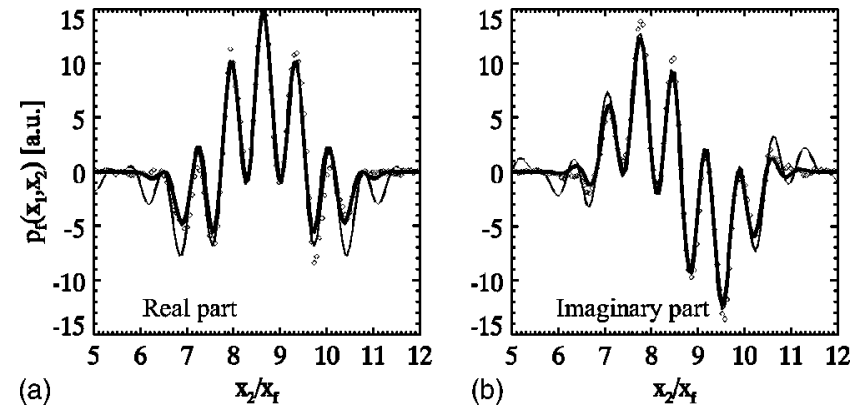

FIG. 2. The reconstruction of the object far-field distribution using the $f$ - $f$ setup in the idler arm. The numerical correlations (open diamonds) were calculated using 2000 pump shots. In (a) and (b) $\psi_{2}^{\mathrm{LO}}$ was chosen as to observe the real and imaginary parts, respectively. The thin line shows $\widetilde{T}_{\text {obj }}$ from Eq. (26), while the thick line is Eq. (13a) as calculated in MATHEMATICA. Slit parameters: $a$ $=9$ pixels, $d=33$ pixels, and $\delta_{x}=23$ pixels, corresponding to 34 , 123 , and $86 \mu \mathrm{m}$, respectively, for $f=5 \mathrm{~cm} . x_{f}=f q_{0} / k_{\mathrm{v}}$.

peated shots of the pump pulse. The optimization steps discussed in the analytical section were carried out (i.e., canceling the quadratic and linear phase terms of the gain). We also present semianalytical calculations performed in MATHEMATICA, which take the analytical formulas from Sec. IV A and carry out the optimization steps and in addition take explicitly into account the finite gain. Thus the MATHEMATICA calculations serve as a bridge between the idealized analytical results (assuming an infinite gain bandwidth) and the numerics (containing finite gain bandwidth and Gaussian pump), in that the MATHEMATICA results take into account the finite gain but still are based on the SPWPA.

The fixed position of the signal detectors $\vec{x}_{1}$ must be chosen with care. In the far-field case we must ensure that in Eq. (13a) the object diffraction pattern $\widetilde{T}_{\text {obj }}\left[\left(\vec{x}_{1}+\vec{x}_{2}\right) k_{v} / f\right]$ is centered where the gain modulus $\left|\gamma\left(-\vec{x}_{2} k_{\mathrm{v}} / f, 0\right)\right|$ has its maximum (which it has at $-\vec{x}_{2} k_{v} / f=-\vec{q}_{C}$ ). Thus we must choose $x_{1} k_{\mathrm{v}} / f=-q_{C}=-8.69$. As for the near-field setup Eq. (20) dictates that also here this position should be used to center the gain over the origin of $\widetilde{T}_{\mathrm{obj}}$. Thus the test arm detector position is unchanged as we pass from the far field to the near field.

Let us first take the case of an $f-f$ setup in the idler arm. Figure 2 displays the two quadrature components corresponding to the real and imaginary parts, confirming that with this setup we can reconstruct the full phase and amplitude information of the object diffraction pattern from the signal-idler correlations. The open diamonds in the figure are results from the numerical simulation, and they are compared to the analytical result (26) shown with a thin line, as well as a MATHEMATICA calculation (thick line) that effectively plots Eq. (13a). Compared to the analytical object distribution, the numerical simulation shows that only the central part of the image is reconstructed since the highfrequency components die out, which is a consequence of the finite gain bandwidth that acts as a low-pass spatial frequency filter. This effect was predicted by Eq. (13a): the MATHEMATICA calculation is based on this equation and it almost coincides with the numerical results. An additional 

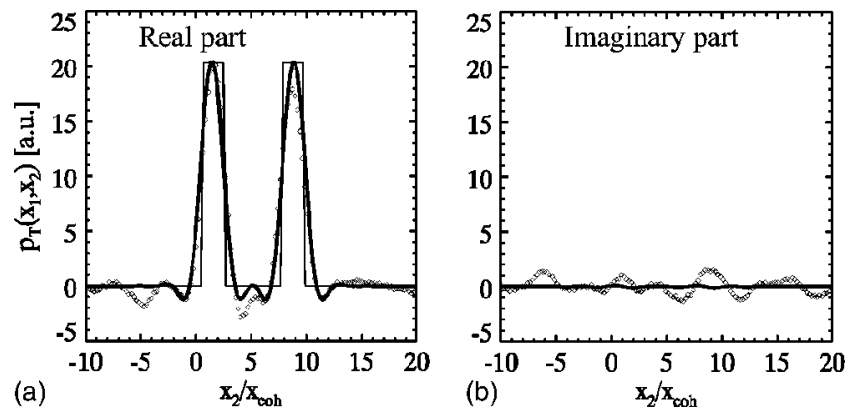

FIG. 3. The reconstruction of the object near-field distribution using the telescope setup in the idler arm. The parameters are identical to Fig. 2, except the thin line here shows $T_{\text {obj }}$ from Eq. (25) swhile the thick line is Eq. (20) as calculated in MATHEMATICA. $x_{\mathrm{coh}}=1 / q_{0}$.

conclusion can be made from this agreement: since the MATHEMATICA result assumes that the pump is a stationary plane wave this implies that the Gaussian profile in space and time of the pump pulse in the numerics does not affect the correlations significantly. This is because the object is in this example very localized so the signal field impinging on it is almost constant. At the end of the section we shall see an example, the pure phase object, where this is not the case.

In Fig. 3 we show that by merely exchanging the $f-f$ setup in the reference arm to the telescope setup as well as properly adjusting the idler LO phase, we can reconstruct the complete object near-field distribution from the signal-idler correlations. Figure 3(a) shows the real part, and the almost zero imaginary part shown in 3(b) confirms that the object does not alter phase information. The analytical SPWPA result using MATHEMATICA is calculated from Eq. (20) [note that it is the integral over $\vec{q}$ that is calculated and not the approximated version given by Eq. (21a)]. In comparison with the analytical object [thin line, Eq. (25)] the numerics are more "blurred," while as before the numerics agree very well with the analytical MATHEMATICA result. It is worthwhile to point out that the results from the near-field and the far-field scenarios are linked: the far field showed a cutoff at high-frequency components due to finite gain. Exactly this cutoff in Fourier space is what gives the blurred character of the near-field image: as predicted by Eq. (20) the correlation is an integral over $\vec{q}$ of the gain multiplied with the object far field, and because the finite gain bandwidth acts as a lowpass filter then it is not possible to reconstruct the sharp edges of the double slit. In other words, the near-field resolution is determined by the far-field bandwidth.

The difference between the two-arm configuration of the ghost-imaging schemes and the Hanbury-Brown-Twiss (HBT) type schemes [31], in which the object is placed in both of the correlated beams, is that the ghost-imaging schemes are able to pertain phase information of the image when reconstructing the diffraction pattern. Indeed, since in the ghost-imaging schemes the object is only placed in one of the beams one may reconstruct the Fourier transform of $T_{\text {obj }}$. This holds both for PDC beams as well as for thermal (or thermal-like) beams [12]. In contrast the HBT schemes either lose or alter phase information since when operated with thermal light the HBT technique can give information
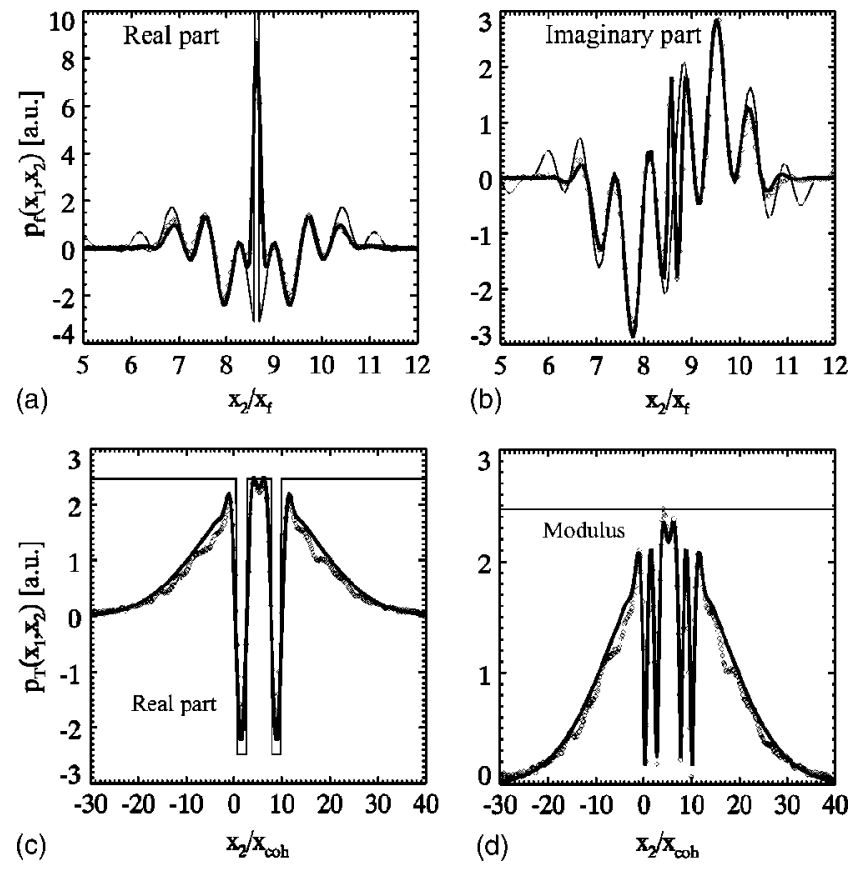

FIG. 4. The reconstruction of a pure phase double slit in (a) and (b) the $f$ - $f$ setup case, and (c) and (d) the telescope case. The parameters are identical to Figs. 2 and 3, except $10^{4}$ shots are used. The MATHEMATICA results (thick) have been adjusted to take into account the finite shape of the signal field.

about the Fourier transform of $\left|T_{\text {obj }}\right|^{2}$, while when operated with PDC beams it can give information about the Fourier transform of $T_{\mathrm{obj}}^{2}[9,12]$.

We show here the results from using the pure phase double slit (27) as an object, since for this object the HBT scheme will not be able to provide information about the diffraction pattern. Figures 4(a) and 4(b) show the far-field reconstruction with the $f-f$ system in the idler arm, and both the real and imaginary parts follow closely the analytical Fourier transform (thin line). Thus the scheme is able to reconstruct the diffraction pattern of a pure phase object. There is a disagreement for the central peak in (a), corresponding to the average value of the real part of the near field: the analytical value actually goes out of the plot range shown. This is due to the fact that ideally the Fourier transform of the chosen phase object has a $\delta$-like behavior here because all the photons are transmitted, whereas in the numerics the signal field impinging on the object has a finite extension. We accommodated for this in the MATHEMATICA calculation by multiplying the phase double slit with a Gaussian fitted to the near-field profile of the signal in the numerics, and as can be seen (thick line) the agreement is now very good. In Figs. 4(c) and 4(d) the near-field reconstruction is shown with the telescope setup in the idler arm, and as evidenced in the real part, (c), the phase object nearfield image can be successfully reconstructed (the imaginary part is not shown since it was roughly zero). Also here the MATHEMATICA calculations include the same finite size of the signal field and match very well with the numerics. We should stress that the blurred shape of the slits is due to the finite gain bandwidth (as in Fig. 3), while the deviation away 
from the slits from the analytical result [thin line, Eq. (27)] is due to the Gaussian profile of the signal [exactly this latter behavior gives rise to a lower central peak in (a)]. Finally, we also show the modulus of the reconstructed near field, (d), to stress that it actually reveals some features of the double slit. This is a consequence of the finite gain bandwidth of the source which turns the signal-idler fields from being completely incoherent (this is the ideal result in the case of infinite bandwidth) to being partially coherent. Note that only with the homodyne detection scheme is it possible to reconstruct the near field of a pure phase object, while with the intensity detection scheme it would not be possible.

\section{Spatial average technique}

In this section we describe how to improve the speed of the correlation convergence as well as to obtain a much larger imaging bandwidth. The idea is to average the correlations not only over repeated shots but also over the position of the signal detector in a certain way. This implies either using a scanning pointlike detector setup or an array of detectors in the test arm, abandoning the idea about keeping the test arm setup as simple as possible. It instead benefits from having a substantially increased bandwidth and convergence rate. It works for reconstructing the far field, but not for the near field because $\Phi_{T}\left(\vec{x}_{1}, \vec{x}_{2}\right)$ depends linearly on $\vec{x}_{1}$, see Eqs. (21b) and (22). Thus it is impossible to engineer the LO phases to observe a given quadrature as $\vec{x}_{1}$ is varied.

For the $f-f$ case, however, the technique works as follows. Let us take the case where we want to see the real part, and assume we have performed all the optimization steps described in Sec. IV A 1. The correlation is then

$$
\begin{aligned}
p_{f}\left(\vec{x}_{1}, \vec{x}_{2}\right)= & 2 T_{d}(\lambda f)^{-2}\left|\gamma\left(-\vec{x}_{2} k_{\mathrm{v}} / f, 0\right) \alpha_{1}\left(\vec{x}_{1}\right) \alpha_{2}\left(\vec{x}_{2}\right)\right| \\
& \times \operatorname{Re}\left\{\widetilde{T}_{\mathrm{obj}}\left[\left(\vec{x}_{1}+\vec{x}_{2}\right) k_{\mathrm{v}} / f\right]\right\} .
\end{aligned}
$$

A change of coordinate system $\vec{x} \equiv \vec{x}_{1}+\vec{x}_{2}$ and averaging over $\vec{x}_{1}$ as $p_{f, \vec{x}_{1}}(\vec{x}) \equiv \int d \vec{x}_{1} p_{f}\left(\vec{x}_{1}, \vec{x}\right)$ gives

$$
\begin{aligned}
p_{f, \vec{x}_{1}}(\vec{x})= & 2 T_{d}(\lambda f)^{-2} \operatorname{Re}\left[\widetilde{T}_{\mathrm{obj}}\left(\vec{x} k_{\mathrm{v}} / f\right)\right] \\
& \times \int d \vec{x}_{1}\left|\gamma\left[\left(\vec{x}_{1}-\vec{x}\right) k_{\mathrm{v}} / f, 0\right] \alpha_{1}\left(\vec{x}_{1}\right) \alpha_{2}\left(\vec{x}-\vec{x}_{1}\right)\right| \\
\simeq & 2 T_{d}(\lambda f)^{-2} \operatorname{Re}\left[\widetilde{T}_{\mathrm{obj}}\left(\vec{x} k_{\mathrm{v}} / f\right)\right]\left|\alpha_{1}(\vec{x})\right| \\
& \times \int d \vec{x}_{1}\left|\gamma\left[\left(\vec{x}_{1}-\vec{x}\right) k_{\mathrm{v}} / f, 0\right] \alpha_{2}\left(\vec{x}-\vec{x}_{1}\right)\right| \\
\simeq & \text { const } \times \operatorname{Re}\left[\widetilde{T}_{\mathrm{obj}}\left(\vec{x} k_{v} / f\right)\right]\left|\alpha_{1}(\vec{x})\right|,
\end{aligned}
$$

where we have assumed that $\left|\alpha_{1}\left(\vec{x}_{1}\right)\right|$ is a slowly varying function and the last approximation holds when $\mid \gamma\left[\left(\vec{x}_{1}\right.\right.$ $\left.-\vec{x}) k_{\mathrm{v}} / f, 0\right] \mid$ is a bound function for all $\vec{x}$. Hence as $\vec{x}$ is scanned the object far field is observed multiplied by the modulus of the signal LO. Thus whereas with $\vec{x}_{1}$ fixed the imaging system had a finite bandwidth, now the bandwidth is effectively only limited by the shape of the signal LO. Therefore if we assume that $\left|\alpha_{1}(\vec{x})\right|$ has a wide enough profile there is no cutoff of the spatial Fourier frequency components; the reconstructed diffraction pattern has a practically infinite bandwidth. We may intuitively understand this by noting the structure of Eq. (28). As we change $\vec{x}_{1}$ to perform the average, the position of the diffraction pattern changes because $p_{f}\left(\vec{x}_{1}, \vec{x}_{2}\right) \propto \operatorname{Re}\left\{\widetilde{T}_{\mathrm{obj}}\left[\left(\vec{x}_{1}+\vec{x}_{2}\right) k_{\mathrm{v}} / f\right]\right\}$. In contrast, the position of the gain remains stationary since $p_{f}\left(\vec{x}_{1}, \vec{x}_{2}\right) \propto\left|\gamma\left(-\vec{x}_{2} k_{\mathrm{v}} / f, 0\right)\right|$. Hence a different part of the diffraction pattern is amplified. Averaging over all $\vec{x}_{1}$ all parts of the diffraction pattern is amplified resulting in a practically unlimited bandwidth. Additionally, the spatial average technique gives an increased convergence rate of the correlation. It is again related to the fact that when the signal detector is changed from $\vec{x}_{1}^{0}$ to $\vec{x}_{1}^{0}$ $+\Delta \vec{x}$ the gain does not change position but the diffraction pattern does. In this case, assuming that $q_{0} f / k_{\mathrm{v}} \gg \Delta x$, the shifted diffraction pattern at $\vec{x}_{1}^{0}+\Delta \vec{x}$ overlaps quite substantially with the previous one. Thus as $\vec{x}_{1}$ is scanned, a given position of the diffraction pattern has many independent contributions within a single shot, thereby increasing the convergence rate.

The possibility of getting rid of the gain cutoff is relying on the object only being located in one arm so the gain is a function of $\vec{x}_{2}$ only while the object far field is a function of $\vec{x}_{1}+\vec{x}_{2}$. In contrast, if the object is located in both arms, as in the HBT scheme, this would not be possible. Notice that this procedure in practice does not amount to merely integrating over $\vec{x}_{1}$ (as when a bucket detector is used). Rather, one should move to position $\vec{x}_{1}$ of the signal detector while moving together the position $\vec{x}_{2}$ of the idler detector as to keep $\vec{x}=\vec{x}_{1}+\vec{x}_{2}$ constant. This corresponds to a spatial convolution between the signal and idler quadratures. To see that, consider the correlation of the measured quadratures $Z_{j}$, and from Eq. (8a) using the substitution $\vec{x}=\vec{x}_{1}+\vec{x}_{2}$ we have $p_{f, \vec{x}_{1}}(\vec{x})=\int_{T_{d}} d t_{1} \int_{T_{d}} d t_{2} \int d \vec{x}_{1}\left\langle Z_{1}\left(\vec{x}_{1}, t_{1}\right) Z_{2}\left(\vec{x}-\vec{x}_{1}, t_{2}\right)\right\rangle$. In the numerical simulations this convolution is rapidly calculated using the fast Fourier transform method.

Note that the bandwidth can also be improved if instead $\vec{x}_{2}$ is fixed and $\vec{x}_{1}$ is scanned. Then, according to Eq. (13a) the gain cutoff is not present, while the diffraction pattern still emerges from the correlations. The numerical simulations confirmed this, however, because no spatial average is performed the convergence rate is as slow as when $\vec{x}_{1}$ is kept fixed. If we average over $\vec{x}_{2}$ we obtain the same result as the spatial average over $\vec{x}_{1}$.

The effects of using the spatial average technique in the same system setup as in Fig. 2 is shown in Fig. 5. First of all, we note the excellent agreement between the numerics and the analytical far-field pattern, and also that there is no frequency cutoff: the imaging bandwidth has become practically infinite. Moreover, the convergence rate has increased substantially compared to that in Fig. 2 (by roughly an order of magnitude). Another interesting point about homodyne detection is that by measuring both quadratures in the the far-field distribution, we may reconstruct the complete nearfield object distribution from this information by using the inverse Fourier transform $F^{-1}[p(\vec{q})]=\int(d \vec{x} / 2 \pi) e^{i \vec{x} \cdot \vec{q}} p(\vec{q})$. We have done this for the data in Figs. 5(a) and 5(b) and the result is shown in Figs. 5(c) and 5(d). The real part, (c), of $F^{-1}\left[p_{f, x_{1}}(x)\right]$ follows $T_{\text {obj }}$ very precisely. This is because we now have access to many more high-frequency components compared to the case shown in Fig. 3. Consequently, as the 

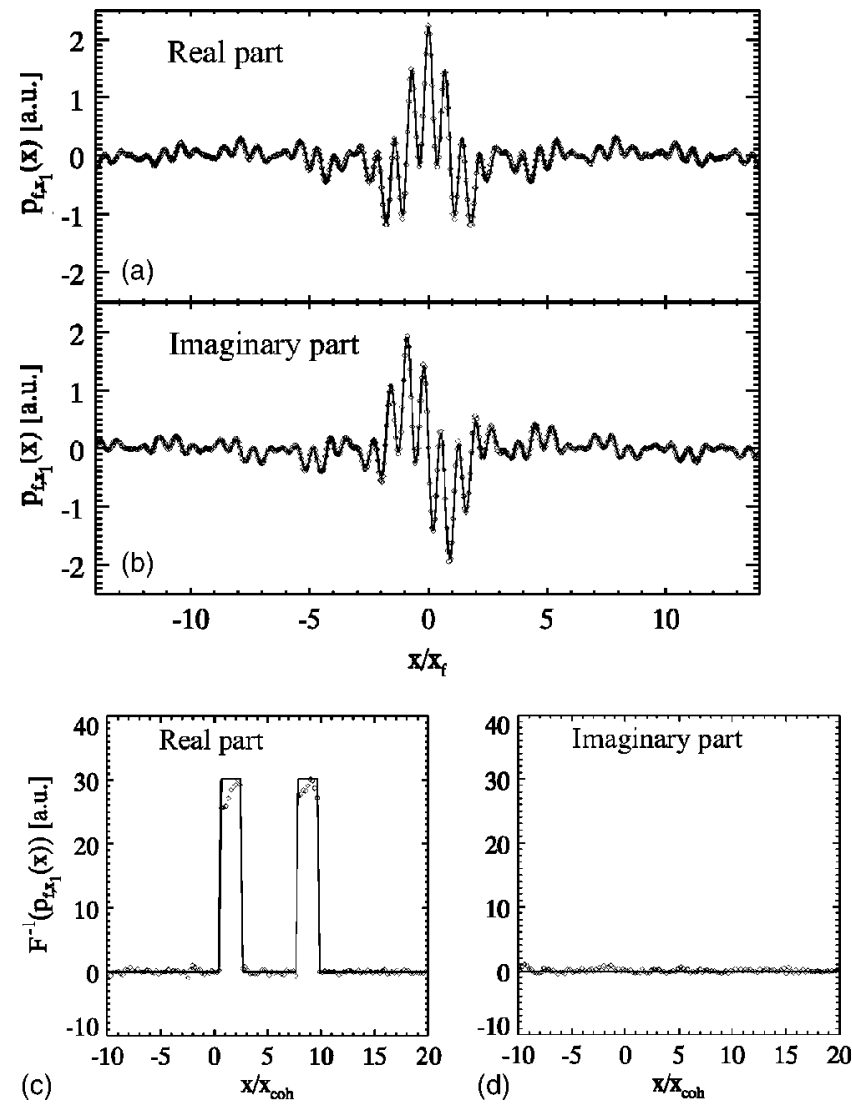

FIG. 5. The same as shown in Fig. 2 except the spatial average technique is used and the average is performed over 200 pump shots. (a) and (b) show $\operatorname{Re}\left\{p_{f, x_{1}}(x)\right\}$ and $\operatorname{Im}\left\{p_{f, x_{1}}(x)\right\}$. Notice that the full numerical grid is shown. (c) and (d) show $\operatorname{Re}\left\{F^{-1}\left[p_{f, x_{1}}(x)\right]\right\}$ and $\operatorname{Im}\left\{F^{-1}\left[p_{f, x_{1}}(x)\right]\right\}$. The thin lines display the analytical results of Eq. (26) for the far field and (25) for the near field.

far-field imaging bandwidth is increased, the near-field resolution improves. It is instructive to note that in the absence of the spatial average the inverse Fourier transform of the farfield correlations shown in Fig. 2 would give exactly the result reported with the telescope setup in Fig. 3. This again underlines the strong link between the near-field and the farfield measurements when all the phase information is intact.

Returning to the phase object of Fig. 4 we repeated the simulations using the spatial average technique to reconstruct the far-field distribution. In Fig. $6 F^{-1}\left[p_{f, x_{1}}(x)\right]$ is shown and the real part now shows excellent agreement with the analytical phase double slit (thin line). Most importantly, Fig. 6(b) showing the modulus now does not reveal any information about the double slit. The extended imaging bandwidth achieved with the spatial average makes the imaging system truly incoherent and hence no phase information is transferred to the modulus.

The spatial average technique is even more superior in two transverse dimensions, since the convergence speedup is increased from averaging also over the additional transverse dimension. In Fig. 7 we used two different objects: (a) an amplitude transmission mask with the letters "INFM," and (b) a more complicated amplitude transmission mask showing a picture of a wolf. We show $\left|F^{-1}\left[p_{f, \vec{x}_{1}}(\vec{x})\right]\right|$ for different
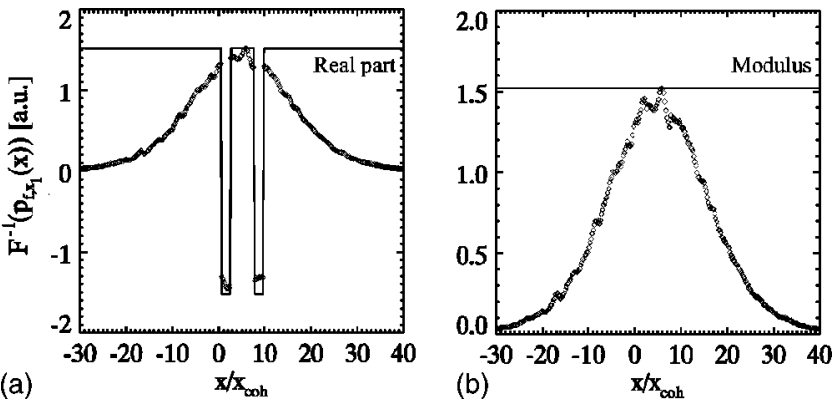

FIG. 6. The same setup as used in Fig. 4 except now the average is also done over $x_{1}$. The far-field correlations are calculated from 1000 repeated pump shots, and (a) and (b) show $\operatorname{Re}\left\{F^{-1}\left[p_{f, x_{1}}(x)\right]\right\}$ and $\left|F^{-1}\left[p_{f, x_{1}}(x)\right]\right|$, respectively.
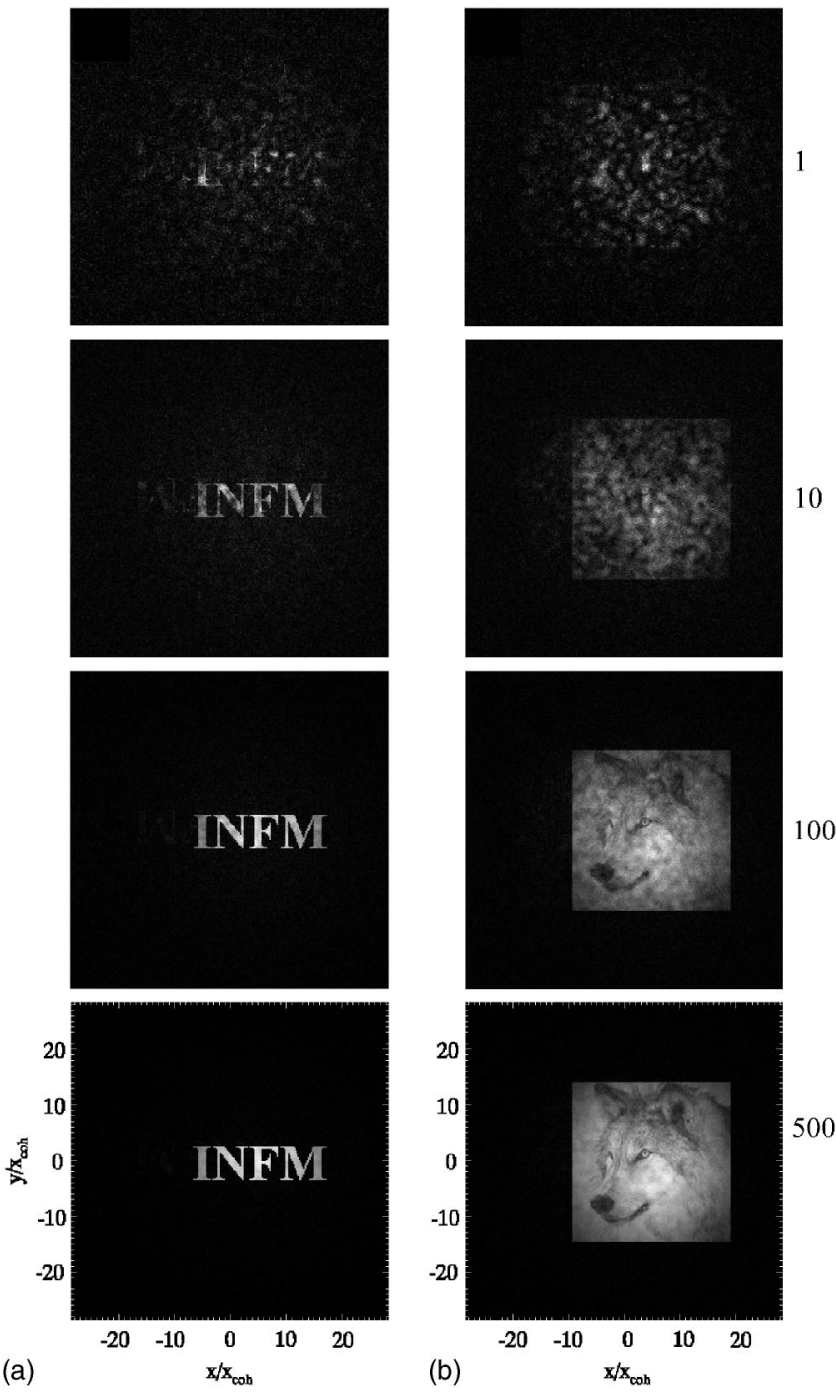

FIG. 7. Using the spatial average technique in an $f$ - $f$ setup with two different objects (a) and (b) to calculate $\left|F^{-1}\left[p_{f, \vec{x}_{1}}(\vec{x})\right]\right|$. The correlations are calculated from a full $(3+1) \mathrm{D}$ simulation, and averaging additionally over the the number of repeated pump shots shown on the right. 

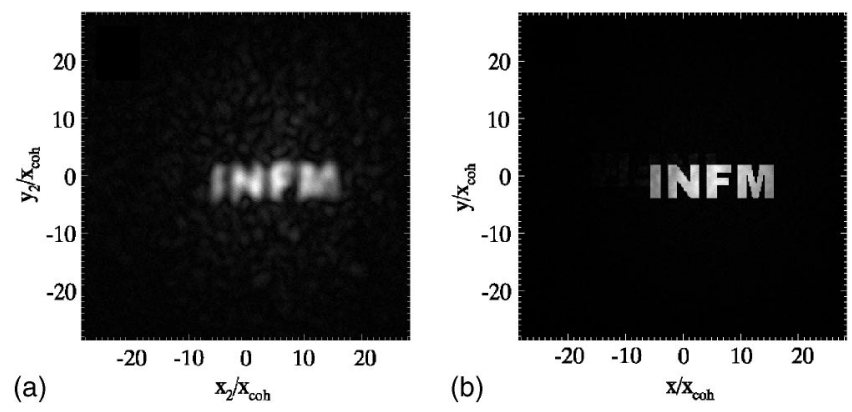

FIG. 8. Qualitative comparison in two dimensions. (a) is the fixed detector case using the telescope setup in the idler arm, and $\left|p_{T}\left(\vec{x}_{1}, \vec{x}_{2}\right)\right|$ is shown after 4000 shots. (b) is the spatial average case using the $f$ - $f$ setup in the idler arm and and $\left|F^{-1}\left[p_{f, \vec{x}_{1}}(\vec{x})\right]\right|$ is shown after 200 shots. The simulations are three-dimensional (3D) in space but neglect the time dimension.

number of shots, and evidently the simple mask (a) converges faster than the more complicated mask (b). Nevertheless, in both cases a good, sharp image is obtained after very few shot repetitions, implying that the corresponding farfield diffraction patterns converge very fast and with a very large bandwidth. After additional averaging over shots (using here 500 shots, as shown in the last frames) the irregularities gradually disappear.

The qualitative difference in two dimensions between the fixed detector case and using the spatial average technique is shown in Fig. 8. (a) displays the modulus of the near-field correlations obtained by using the telescope setup and a fixed signal detector position, and (b) displays the modulus of the inverse Fourier transform of the far-field correlations obtained using the $f$ - $f$ setup and applying the spatial average technique. The blurred character in (a) using fixed detectors is obvious (similar to what was seen in one transverse dimension, see, e.g., Fig. 3), and does not improve much even if more averages are performed since it is a consequence of the finite bandwidth. In contrast the much improved far-field bandwidth of the spatial average technique in (b) increases the near-field resolution so the observed near-field image is completely sharp. Moreover, only 200 shots is used in (b) in contrast to the 4000 in (a). Hence the convergence rate in two dimensions using the spatial average technique is increased with roughly two orders of magnitude when compared to the fixed detector case. Note that the simulations in Fig. 8 have been performed neglecting the time dimension in order to save CPU time. Such an approximation corresponds to using a narrow interference filter, and was justified by comparing with results for a fewer number of shots including also time. A further justification of this is that, as is pointed out in Appendix B, when $T_{d} \gg \tau_{\text {coh }}$ the overlap of the LO with the field generally tends to select the $\Omega=0$ component corresponding to neglecting time (except for a LO duration shorter that $\left.\tau_{\mathrm{coh}}\right)$.

We have in this section shown that using pointlike detectors in the test arm allows us to reconstruct both amplitude and phase information about an image, with the near-field and far-field object distributions accessible by merely changing the optical setup of the reference arm. The spatial imaging bandwidth for a fixed test detector position is determined by the source bandwidth, and this in turn determines the near-field resolution. Using a spatial average technique improves the imaging bandwidth dramatically in the far-field case, and also leads to a much faster information retrieval.

\section{BUCKET DETECTORS IN TEST ARM}

We now turn to the case where bucket detectors are used in the test arm, a setup used previously with the intensity detection scheme to observe the object near field $[4,14]$. One motivation is to show that by using homodyne detection together with bucket detectors, phase-only objects can be imaged. If an intensity detection scheme is used this is not possible, as shown in Ref. [14] for the two-photon coincidence imaging case (although it is possible using a pointlike detector in the object arm $[8,10])$. This is a general result that also holds in the high-gain regime (it can easily be derived from the results of Refs. [11,12]). Another motivation is to see the imaging capabilities of this setup since it technically seems simpler than the pointlike detector setup.

As argued in Sec. III, when bucket detectors are used in the test arm it is necessary to keep the object placed in the measurement plane. Besides this, the lens setups in the arms are arbitrary, but we decided to keep the $f-f$ setup in the signal arm, as well as the idler setup in either the $f$ - $f$ or the telescope configuration. The test-arm setup is shown in Fig. $1(\mathrm{c})$.

\section{A. Analytical results}

The test arm in the bucket detector case has the kernel (5b), so to evaluate the sanalytical form of the quadrature correlation (12) we must use it in the form (11b). Then, as we will now show, using the idler kernels (6a) and (6b) we can obtain the near-field and the far-field distributions, respectively. The bucket detectors in the test arm effectively integrate over $\vec{x}_{1}$ of the quadrature correlation giving $\bar{p}_{T}^{\mathrm{b}}\left(\vec{x}_{2}\right)$ $\equiv \int d \vec{x}_{1} p_{T}^{\mathrm{b}}\left(\vec{x}_{1}, \vec{x}_{2}\right)$

\section{Retrieval of object near field}

We here use the $f$ - $f$ setup in the reference arm, implying we must use the idler kernel Eq. (6a) in the form (11b). Inserting this in Eq. (12) and integrating over $\vec{x}_{1}$ the signalidler quadrature correlation for retrieving the object near field is consequently

$$
\begin{gathered}
\bar{p}_{f}^{\mathrm{b}}\left(\vec{x}_{2}\right)=\frac{T_{d}}{\pi}\left|\alpha_{1}\left(-\vec{x}_{2}\right) \alpha_{2}\left(\vec{x}_{2}\right) \gamma\left(-\vec{x}_{2} k_{\mathrm{v}} / f, 0\right)\right| \\
\times \operatorname{Re}\left[T_{\mathrm{obj}}\left(-\vec{x}_{2}\right) e^{\bar{\Phi}_{f}^{b}\left(\vec{x}_{2}\right)}\right] \\
\bar{\Phi}_{f}^{\mathrm{b}}\left(\vec{x}_{2}\right) \equiv \phi_{\gamma}\left(-\vec{x}_{2} k_{\mathrm{v}} / f, 0\right)-\phi_{1}^{\mathrm{LO}}\left(-\vec{x}_{2}\right)-\phi_{2, f}^{\mathrm{LO}}\left(\vec{x}_{2}\right)+\pi
\end{gathered}
$$

The idler LO phase may then be engineered to observe the desired quadrature and as a first approximation the gain phase dependence is neglected, so to observe the real part we must choose 


$$
\psi_{2, f}^{\mathrm{LO}}=\phi_{\gamma}\left(-\vec{q}_{C}, 0\right)-\phi_{1}^{\mathrm{LO}}\left(-\vec{x}_{2}\right)+\pi .
$$

We may optimize this result by setting the focus plane of the $f$ - $f$ setup in the idler arm inside the crystal with the amount given by Eq. (18) as to compensate the quadratic term of the gain phase. Additionally, by making the idler LO a tilted wave with the wave number given by Eq. (17) we compensate for the linear phase term. With these optimizations, the real part can be observed by choosing

$$
\psi_{2, f}^{\mathrm{LO}}=\phi_{\gamma}^{(0)}-\phi_{1}^{\mathrm{LO}}\left(-\vec{x}_{2}\right)+\pi
$$

We note that the results show a dependence of the signal LO as $\alpha_{1}\left(-\vec{x}_{2}\right)$. Therefore as the idler pixels are scanned it is crucial that the signal LO does not vary substantially. Another point is the scale of the coordinate system, but we will return to this in the next subsection.

\section{Retrieval of object far field}

For a telescope setup in the reference arm we must use the kernel (6b) in the form (11b). Inserting this in Eq. (12) and integrating over $\vec{x}_{1}$ the measured correlation is

$$
\begin{aligned}
\bar{p}_{T}^{\mathrm{b}}\left(\vec{x}_{2}\right)= & \frac{T_{d}}{i \lambda f} \alpha_{2}^{*}\left(\vec{x}_{2}\right) \\
& \times \int \frac{d \vec{x}_{1}}{2 \pi} e^{-i \vec{x}_{1} \cdot \vec{x}_{2} k_{\mathrm{v}} / f} \gamma\left(\vec{x}_{1} k_{\mathrm{v}} / f, 0\right) \alpha_{1}^{*}\left(\vec{x}_{1}\right) T_{\mathrm{obj}}\left(\vec{x}_{1}\right)+\text { c.c. }
\end{aligned}
$$

We assume now that the plateau-shaped gain and the modulus of the signal LO vary slowly with respect to the object, so they can be taken out of the integral, evaluated at the position of maximum gain $\vec{x}_{1} k_{\mathrm{v}} / f=-\vec{q}_{C}$. This approximation gives the quadrature correlation for observing the object far field,

$$
\begin{aligned}
\bar{p}_{T}^{\mathrm{b}}\left(\vec{x}_{2}\right) \simeq & \frac{2 T_{d}}{\lambda f}\left|\gamma\left(-\vec{q}_{C}, 0\right) \alpha_{1}\left(-\vec{q}_{C} f / k_{\mathrm{v}}\right) \alpha_{2}\left(\vec{x}_{2}\right)\right| \\
& \times \operatorname{Re}\left[\tilde{T}_{\mathrm{obj}}\left(\vec{x}_{2} k_{\mathrm{v}} / f+\vec{q}_{1}^{\mathrm{LO}}\right) e^{i \bar{\Phi}_{T}^{\mathrm{b}}\left(\vec{x}_{2}\right)}\right], \\
\bar{\Phi}_{T}^{\mathrm{b}}\left(\vec{x}_{2}\right) \equiv & \phi_{\gamma}\left(-\vec{q}_{C}, 0\right)-\psi_{1}^{\mathrm{LO}}-\phi_{2, T}^{\mathrm{LO}}\left(\vec{x}_{2}\right)+\pi / 2 .
\end{aligned}
$$

Assuming a constant gain phase the real part of the object far field can be observed if

$$
\phi_{2, T}^{\mathrm{LO}}\left(\vec{x}_{2}\right)=\phi_{\gamma}\left(-\vec{q}_{C}, 0\right)-\psi_{1}^{\mathrm{LO}}+\pi / 2 .
$$

As in the other cases the result (34a) can be optimized by compensating for the quadratic gain phase term before the integration by taking the imaging plane of the telescope setup inside the crystal with the amount given by Eq. (18). Thus in the optimized case to observe the real part of the far field the reference phase is

$$
\phi_{2, T}^{\mathrm{LO}}\left(\vec{x}_{2}\right)=\phi_{\gamma}^{(0)}-\psi_{1}^{\mathrm{LO}}+\pi / 2 .
$$

In connection with optimizing the imaging performance, it is apparent from Eq. (34a) that if the signal LO is a tilted wave then the origin of the reconstructed diffraction pattern changes. This can be used to compensate for undesired walk-
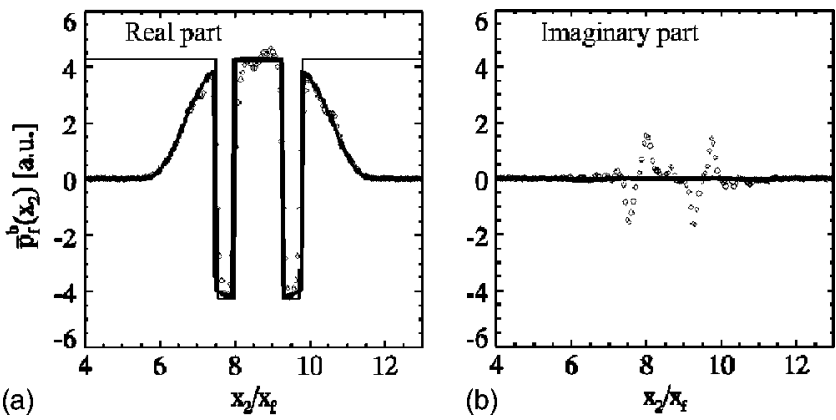

FIG. 9. The reconstruction of the object near-field distribution of a pure phase object with bucket detectors in the test arm and using the $f$ - $f$ setup in the reference arm. The numerical correlations (open diamonds) were calculated from averaging over $10^{4}$ pump shots. The thin line is the pure phase double slit (27), while the thick line is calculated in MATHEMATICA on basis of Eq. (30a). Slit parameters in pixels as Fig. 2 corresponding for $f=5 \mathrm{~cm}$ to $a=166 \mu \mathrm{m}, d$ $=610 \mu \mathrm{m}$, and $\delta x=-2.9 \mathrm{~mm}$.

off effects by centering the reconstructed image in the place where the idler (which in this setup is in the near field) has its maximum. Additionally, making the idler LO a tilted wave can be used to compensate for unimportant oscillations in the quadrature components that comes from shifting the object near field from origin [see in that connection Eq. (26)].

Let us come back to the scaling of the image information. The particular setup we chose (having the object placed in the far field created by an $f$ - $f$ lens system, just before the bucket detectors) implies that the signal field impinging on the object changes on the scale $x_{f}=f q_{0} / k_{\mathrm{v}}$. This is evident in the analytical formulas Eqs. (30a) and (33). In contrast, when the object was placed in the near field the field changed on the scale $x_{\text {coh }}=1 / q_{0}$. As an example, for $f=5 \mathrm{~cm}$ and $\lambda$ $=704 \mathrm{~nm}, x_{f}=338 \mu \mathrm{m}$ while in contrast $x_{\text {coh }}=17 \mu \mathrm{m}$. The consequence is that while imaging is possible with bucket detectors, it occurs on a different (i.e., larger) length scale than with pointlike detectors.

\section{B. Numerical results}

The numerical simulations using bucket detectors were similar to those presented in Sec. IV B, except now the object is placed after the $f$ - $f$ setup in the test arm. We decided to keep the object structure used in Fig. 4, i.e., a pure phase double slit with exactly the same number of pixels between the slits as well as for the slit aperture. Hence the physical slit dimensions are determined by the larger scale $x_{f}$. In addition, all the optimization procedures discussed in the previous sections were used.

Figure 9 shows the numerical simulation using the $f$ - $f$ setup in the idler arm. The reconstructed real part in (a) follows the analytical double slit, while the imaginary part (b) is containing only little information about the slits. Consequently using bucket detectors in the test arm both phase and amplitude information about the object near field can be reconstructed. Notice that the MATHEMATICA result (thick line) has a better resolution than the numerical results. This 

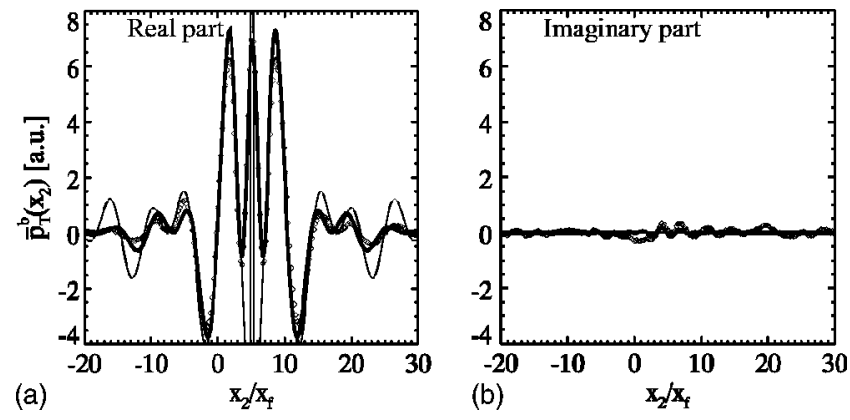

FIG. 10. The same as shown in Fig. 9 except now using the telescope setup in the idler arm making it possible to reconstruct the object far-field distribution. The thick line is Eq. (33) calculated in MATHEMATICA while taking into account the finite shape of the idler near-field distribution.

indicates that the finite shape of the pump worsens the resolution, and we will come back to this later. Away from the slits the correlations decay to zero because of the finite shape of the far-field gain.

Figure 10 shows a numerical simulation similar to Fig. 9 but with the telescope setup in the idler arm. The reconstructed real part in (a) follows within a certain bandwidth the analytical result (thin line) while the imaginary part (b) is zero. Thus using bucket detectors in the test arm both phase and amplitude information about the object far-field distribution can be reconstructed. The cutoff in (a) is actually caused by the Gaussian shape of the idler near-field profile, which has been taken into account in the MATHEMATICA calculation of the integral (33); without such a correction the MATHEMATICA result would actually very closely follow the analytical result (thin line) without being cut off. This can be understood by inspecting Eq. (33) more closely, since we see that the action of the gain inside the integral is to provide a limit for the object extension. However, in contrast to the pointlike detector case of Eq. (13a) where the gain eventually determines the far-field imaging bandwidth, in the bucket case it is not so: Once the object is located inside the gain, the reproduced diffraction pattern is very good. This is also in accord with what we saw in the semianalytical reproduction of the near field in Fig. 9, where the double slits appear very sharp indicating that the Fourier frequency bandwidth is large. A consequence of this is that if we tailor the pump to have a plane-wave shape, we should see practically no cutoff in the far field as well as a very sharp reproduction of the double slit in the near field. We checked this to be the case. Thus also the bucket detector case shows an inherent link between far-field bandwidth and near-field resolution. Note that in (b) $\operatorname{Im}\left[\bar{p}_{T}^{\mathrm{b}}\left(x_{2}\right)\right] \simeq 0$ because we used a tilted-wave idler LO in order to eliminate the oscillations arising because of the offset of the object from origin, making the Fourier transform purely real. Additionally, the central peak of the analytical function in (a) goes out of the range shown for the same reasons as in Fig. 4.

To conclude this section, a homodyne detection scheme with bucket detectors in the test arm is capable of observing both amplitude and phase distribution of an object. Both the near-field and the far-field distributions are accessible by only changing the optical setup in the reference arm and even phase-only objects can be imaged with a bucket detector, in contrast to the intensity detection case [14]. We finally note that only with the homodyne technique is it possible to reconstruct the diffraction pattern of an object using bucket detectors in the test arm. And while the intensity detection case may reproduce the object near field using a bucket detector in the test arm [4,14,32], as mentioned above only the modulus of the object near field may be reproduced.

\section{CONCLUSION}

We have shown that homodyne detection can be used to get access to both modulus and phase information in ghostimaging schemes based on PDC in the high-gain regime. Both the signal and idler beams are measured with individual LO's, and we have shown analytically how to engineer the phases of these in order to retrieve amplitude as well as phase information of the object image (near field) and the object diffraction pattern (far field). Generally, the results indicate that merely fixing a reference phase of the idler LO is enough to generate the desired quadrature correlations, except for the near field that in addition requires a tilted wave LO. An improvement can in all cases be achieved if the quadratic dependence of gain phase is compensated for, primarily by keeping the focusing plane a short distance inside the crystal. Interestingly, even when using bucket detectors in the test arm the homodyne technique could completely reconstruct both the amplitude and phase of an image. The results were confirmed with numerical simulations taking into account a finite shape of the pump pulse.

We showed that when reconstructing the diffraction pattern a spatial average over the signal detector position leads to much faster convergence rate of the correlations (fewer pump-shot repetitions needed) as well as a hugely improved bandwidth of the diffraction pattern. An intuitive explanation of the method is found from Eq. (28): as the signal detector position is changed to perform the average, the position of the diffraction pattern in the correlations is also changed. In contrast, the position of the gain is not changing since it only depends on the position of the idler detector. Hence as the signal detector position is changed, different parts of the diffraction pattern is amplified leading to a larger bandwidth. The improved convergence rate is related to the fact that within a single shot there are many independent modes that each contribute to the correlation statistics. Thus the spatial average technique truly exploits the possibility for parallel operations in spatially correlated beams. The technique cannot be applied in the near-field case, but it is important to note that the technique works when reconstructing the diffraction pattern using direct intensity measurements of the fields, both when using PDC beams (as will be discussed elsewhere in a separate publication [32]) and the thermal-like beams investigated in Ref. [12]. The technique works particularly well in the high-gain regime because the number of signal photons per mode is not small, but should also work in the low-gain regime. Finally, it is worthwhile to mention that when using bucket detectors in the test arm the imaging bandwidth was determined by the finite shape of the pump. Hence to improve the bandwidth a pump with a larger waist should be chosen. 
Since the homodyne scheme allows us to have access to both phase and amplitude information, we may pass from the far-field result to the near-field result by simply making an inverse Fourier transform. Thus by taking the far-field correlations containing the information about the diffraction pattern and performing an inverse Fourier transform gave the same result as measuring the near-field correlations containing the information about the object image, and vice versa. An important consequence is that we can exploit the spatial average technique to improve the bandwidth of the reconstructed diffraction pattern, and through an inverse Fourier transform obtain an image with a hugely improved resolution. This is not possible in the intensity detection case.

In the time domain the outcome of a homodyne detection is depending strongly on the temporal overlap between the local oscillator and the field. A question to answer in this paper was therefore how important it is to ensure a proper overlap also in the spatial domain between the local oscillator and the field. The answer in the context of ghost imaging is that it is not crucial. In fact, the analytical results in the plane-wave pump approximation suggest that the near-field shape of the local oscillator is merely multiplied onto the final result. Even the numerical simulations taking a Gaussian shape of the fields into account suggest the same. Another issue entirely is measurements where the quantum efficiency is important (in contrast to here), and in that case undoubtably the spatial overlap becomes more important.

Using an intensity detection scheme the detection time must not be larger than the coherence time, otherwise the background term that contains no image information becomes dominating and the visibility of the image is dramatically decreased $[7,12,13]$. In contrast, the homodyne detection scheme is not restricted by this because the correlation function is second order in the field operators implying there is no background term. Hence the detection time may be chosen much larger than the coherence time. Additionally, we have shown that the spatial average technique can give a much larger imaging bandwidth than the source itself. This suggests the possibility of using an optical parametric oscillator (OPO) as a source, and using homodyne detection as a measurement protocol. The limited spatial bandwidth of the OPO with respect to PDC could then be circumvented with the spatial average technique, and the homodyne measurements allow a continuous wave operation of the imaging scheme: since there are no problems with image visibility, the measurement time can be taken longer than the coherence time. Thus one could effectively abandon the involved scheme of using a high-power laser pumping a PDC setup and the complicated task of overlapping LO fields with the signal-idler fields with temporal durations on a ps level.

\section{ACKNOWLEDGMENTS}

We are grateful to Prem Kumar and Claude Fabre for stimulating discussions. This project has been carried out within the framework of the FET project QUANTIM of the EU, of the PRIN project MIUR "Theoretical Study of Novel Devices Based on Quantum Entanglement," and of the INTAS project "Non-classical Light in Quantum Imaging and
Continuous Variable Quantum Channels." M.B. acknowledges financial support from the Danish Technical Research Council (STVF) and the Carlsberg Foundation.

\section{APPENDIX A: GAIN FUNCTIONS AND THE EXPANSION OF THE GAIN PHASE}

The gain functions of Eq. (3) are for the signal [20]

$$
\begin{aligned}
U_{1}(\vec{q}, \Omega)= & e^{i D_{12}(\vec{q}, \Omega) l_{c} / 2}\left[\cosh \left(\Gamma_{12}(\vec{q}, \Omega) l_{c}\right)\right. \\
& \left.+i \frac{\Delta_{12}(\vec{q}, \Omega)}{2 \Gamma_{12}(\vec{q}, \Omega)} \sinh \left(\Gamma_{12}(\vec{q}, \Omega) l_{c}\right)\right], \\
V_{1}(\vec{q}, \Omega)= & e^{i D_{12}(\vec{q}, \Omega) l_{c} / 2} \frac{\sigma_{p} \sinh \left[\Gamma_{12}(\vec{q}, \Omega) l_{c}\right]}{\Gamma_{12}(\vec{q}, \Omega)} .
\end{aligned}
$$

For the idler similar gain functions are found by exchanging indices $1 \leftrightarrow 2$. We have in Eq. (A1) introduced

$$
\Delta_{i j}(\vec{q}, \Omega) \equiv \Delta_{0}+\delta_{i}(\vec{q}, \Omega)+\delta_{j}(-\vec{q},-\Omega),
$$

as well as $D_{i j}(\vec{q}, \Omega) \equiv \delta_{i}(\vec{q}, \Omega)-\delta_{j}(-\vec{q},-\Omega)-\Delta_{0}, \quad \Gamma_{i j}(\vec{q}, \Omega)$ $\equiv \sqrt{\sigma_{p}^{2}-\left[\Delta_{i j}(\vec{q}, \Omega) / 2\right]^{2}}$ and $\sigma_{p} \equiv \sigma A_{p}$.

When the signal and idler input fields of the crystal are in the vacuum state the output fields are [20]

$$
\begin{aligned}
& \left\langle a_{j}^{\text {out } \dagger}(\vec{q}, \Omega) a_{k}^{\text {out }}\left(\vec{q}^{\prime}, \Omega^{\prime}\right)\right\rangle \\
& \quad=\delta_{j k} \delta\left(\vec{q}-\vec{q}^{\prime}\right) \delta\left(\Omega-\Omega^{\prime}\right)\left|V_{j}(\vec{q}, \Omega)\right|^{2}, \quad j=1,2, \\
& \left\langle a_{1}^{\text {out }}(\vec{q}, \Omega) a_{2}^{\text {out }}\left(\vec{q}^{\prime}, \Omega^{\prime}\right)\right\rangle \\
& \quad=\delta\left(\vec{q}+\vec{q}^{\prime}\right) \delta\left(\Omega+\Omega^{\prime}\right) U_{1}(\vec{q}, \Omega) V_{2}(-\vec{q},-\Omega) .
\end{aligned}
$$

The bandwidths of emission of the gain functions (A1) in the spatial and temporal frequency domain are

$$
\begin{gathered}
q_{0}^{2}=2\left[l_{c}\left(1 / k_{1}+1 / k_{2}\right)\right]^{-1}, \\
\Omega_{0}=\left(\left|k_{1}^{\prime}-k_{2}^{\prime}\right| l_{c}\right)^{-1},
\end{gathered}
$$

where the coherence length and coherence time describe the typical variation scales of the signal-idler fields:

$$
x_{\mathrm{coh}}=1 / q_{0}, \quad \tau_{\mathrm{coh}}=1 / \Omega_{0} .
$$

It is useful to perform a quadratic expansion for the gain phase $\phi_{\gamma}$ from the expressions (A1). We note that $\Delta_{12}(\vec{q}, \Omega)=\Delta_{21}(-\vec{q},-\Omega) \equiv \Delta(\vec{q}, \Omega)$ and therefore also $\Gamma_{12}(\vec{q}, \Omega)=\Gamma_{21}(-\vec{q},-\Omega) \equiv \Gamma(\vec{q}, \Omega)$. Thus we obtain

$$
\begin{aligned}
\phi_{\gamma}(\vec{q}, \Omega)= & -\Delta_{0} l_{c}+\arctan \left[\frac{\Delta(\vec{q}, \Omega) \tanh \left[\Gamma(q, \Omega) l_{c}\right]}{2 \Gamma(\vec{q}, \Omega)}\right] \simeq-\Delta_{0} l_{c} \\
& +\arctan \left[\Psi_{\gamma} \Delta(\vec{q}, \Omega) l_{c}\right] \simeq-\Delta_{0} l_{c}+\Psi_{\gamma} \Delta(\vec{q}, \Omega) l_{c},
\end{aligned}
$$

where we use that the gain is large and that the modes inside 
the gain are phase matched $[\Delta(\vec{q}, \Omega) \simeq 0]$ making $\Gamma(\vec{q}, \Omega)$ $\simeq \sigma_{p}$. We have introduced a dimensionless parameter that is related to the dimensionless gain $\sigma_{p} l_{c}$,

$$
\Psi_{\gamma} \equiv \frac{\tanh \left(\sigma_{p} l_{c}\right)}{2 \sigma_{p} l_{c}} .
$$

For $\sigma_{p} l_{c} \rightarrow 0$ (small gain) $\Psi_{\gamma} \rightarrow 1 / 2$ while for $\sigma_{p} l_{c} \gg 1$ (large gain) $\Psi_{\gamma} \rightarrow 0$. Now from Eq. (A2) it is evident that $\Delta(\vec{q}, \Omega)$ is quadratic in $\vec{q}$ and $\Omega$, so that the gain phase (A6) approximately can be written as

$$
\begin{gathered}
\phi_{\gamma}^{(0)}=\Delta_{0} l_{c}\left(-1+\Psi_{\gamma}\right), \\
\vec{\phi}_{\gamma, q}^{(1)}=-\rho_{2} l_{c} \Psi_{\gamma} \vec{e}_{x}, \\
\phi_{\gamma, q}^{(2)}=-\frac{\left(n_{1}+n_{2}\right) l_{c} \Psi_{\gamma}}{2 n_{1} n_{2} k_{\mathrm{v}}}, \\
\phi_{\gamma, \Omega}^{(1)}=\left(k_{1}^{\prime}-k_{2}^{\prime}\right) l_{c} \Psi_{\gamma}, \\
\phi_{\gamma, \Omega}^{(2)}=\left(k_{1}^{\prime \prime}+k_{2}^{\prime \prime}\right) l_{c} \Psi_{\gamma} / 2,
\end{gathered}
$$$$
\phi_{\gamma}(\vec{q}, \Omega) \simeq \phi_{\gamma}^{(0)}+\vec{\phi}_{\gamma, q}^{(1)} \cdot \vec{q}+\phi_{\gamma, q}^{(2)}|\vec{q}|^{2}+\phi_{\gamma, \Omega}^{(1)} \Omega+\phi_{\gamma, \Omega}^{(2)} \Omega^{2},
$$

where $\vec{e}_{x}$ is a unit vector in the $x$ direction.

\section{APPENDIX B: SIGNAL-IDLER CORRELATION}

Here we present the detailed calculations of the expressions for the signal-idler correlation (8b). By using Eq. (11a) we see that in Eq. (8b) the Fourier spatial and temporal frequencies are decoupled as

$$
\begin{aligned}
p\left(\vec{x}_{1}, \vec{x}_{2}\right)= & \int d \Omega B\left(\vec{x}_{1}, \vec{x}_{2}, \Omega\right) \\
& \times \int d \vec{q} h_{1}\left(\vec{x}_{1},-\vec{q}\right) h_{2}\left(\vec{x}_{2}, \vec{q}\right) \gamma(\vec{q}, \Omega)+\text { c.c. }, \\
B\left(\vec{x}_{1}, \vec{x}_{2}, \Omega\right) \equiv & T_{d}^{2} \iint \frac{d \Omega^{\prime} d \Omega^{\prime \prime}}{(2 \pi)^{2}} \alpha_{1}^{*}\left(\vec{x}_{1}, \Omega^{\prime}\right) \alpha_{2}^{*}\left(\vec{x}_{2}, \Omega^{\prime \prime}\right) \\
& \times \operatorname{sinc}\left[\left(\Omega-\Omega^{\prime}\right) T_{d} / 2\right] \operatorname{sinc}\left[\left(\Omega+\Omega^{\prime \prime}\right) T_{d} / 2\right] .
\end{aligned}
$$

(B1b)
Let us consider the case of a pulsed LO, i.e., evaluate Eq. (B1b) for the LO duration much smaller than $\tau_{\text {coh }}$. Additionally it can be assumed that $T_{d} \gg \tau_{\text {coh }}$ implying $T_{d} \operatorname{sinc}\left[\left(\Omega \pm \Omega^{\prime}\right) T_{d} / 2\right] \simeq 2 \pi \delta\left(\Omega \pm \Omega^{\prime}\right), \quad$ giving $B\left(\vec{x}_{1}, \vec{x}_{2}, \Omega\right)$ $=\alpha_{1}^{*}\left(\vec{x}_{1}, \Omega\right) \alpha_{2}^{*}\left(\vec{x}_{2}, \Omega\right)$, which means that the correlation is

$$
\begin{aligned}
p\left(\vec{x}_{1}, \vec{x}_{2}\right)= & \int d \Omega \alpha_{1}^{*}\left(\vec{x}_{1}, \Omega\right) \alpha_{2}^{*}\left(\vec{x}_{2}, \Omega\right) \\
& \times \int d \vec{q} h_{1}\left(\vec{x}_{1},-\vec{q}\right) h_{2}\left(\vec{x}_{2}, \vec{q}\right) \gamma(\vec{q}, \Omega)+\text { c.c. }
\end{aligned}
$$

In the case of a continuous wave $\mathrm{LO}$, the $\mathrm{LO}$ duration is much longer that $\tau_{\mathrm{coh}}$. Thus the LO corresponds to a quasimonochromatic wave, i.e., $\alpha_{j}(\vec{x}, \Omega)=\delta(\Omega) \alpha_{j}(\vec{x})$. Using this form in Eq. (B1b) removes the integration on $\Omega^{\prime}$ and $\Omega^{\prime \prime}$. Then we use that for $T_{d} \gg \tau_{\text {coh }}$ the remaining term $T_{d}\left[\operatorname{sinc}\left(\Omega T_{d} / 2\right)\right]^{2} \quad$ behaves like $2 \pi \delta(\Omega)$, implying $B\left(\vec{x}_{1}, \vec{x}_{2}, \Omega\right) \simeq \delta(\Omega) \alpha_{1}^{*}\left(\vec{x}_{1}\right) \alpha_{2}^{*}\left(\vec{x}_{2}\right) T_{d} /(2 \pi)$. Hence

$$
\begin{aligned}
p\left(\vec{x}_{1}, \vec{x}_{2}\right) \simeq & \frac{T_{d}}{2 \pi} \alpha_{1}^{*}\left(\vec{x}_{1}\right) \alpha_{2}^{*}\left(\vec{x}_{2}\right) \\
& \times \int d \vec{q} h_{1}\left(\vec{x}_{1},-\vec{q}\right) h_{2}\left(\vec{x}_{2}, \vec{q}\right) \gamma(\vec{q}, 0)+\text { c.c. }
\end{aligned}
$$

Clearly, the pulsed case may have a larger gain than the monochromatic case because of the integration over $\Omega$, but only if we can minimize the $\Omega$ dependence of the phase in the integrand of Eq. (B2), which will in turn maximize the integral regardless of the value of $\vec{q}$. As an approximation, a proper temporal delay between the LO's effectively cancels the first-order term (A8e) of the gain phase. It is not easy to cancel the second-order term, but we estimate that this term does not contribute much since $\left(k_{1}^{\prime \prime}+k_{2}^{\prime \prime}\right) l_{c} \Omega_{0}^{2} / 2=\left(k_{1}^{\prime \prime}\right.$ $\left.+k_{2}^{\prime \prime}\right) /\left[\left(k_{2}^{\prime}-k_{1}^{\prime}\right)^{2} l_{c}\right]=O\left(10^{-4}\right)$ for the crystal setup we consider. The numerics showed only a slightly enlarged effective spatial bandwidth due to the delay between the LO's, and since it requires temporally short pulses (roughly less than $20 \%$ of $\tau_{\text {coh }}$ ) which are difficult to obtain experimentally, we used the limit of a continuous wave LO for the analytical results.
[1] D. N. Klyshko, Zh. Eksp. Teor. Fiz. 94, 82 (1988) [Sov. Phys. JETP 67, 1131 (1988)].

[2] A. V. Belinskii and D. N. Klyshko, Zh. Eksp. Teor. Fiz. 105, 487 (1994) [JETP 78, 259 (1994)].

[3] D. V. Strekalov, A. V. Sergienko, D. N. Klyshko, and Y. H. Shih, Phys. Rev. Lett. 74, 3600 (1995).

[4] T. B. Pittman, Y. H. Shih, D. V. Strekalov, and A. V. Ser- gienko, Phys. Rev. A 52, R3429 (1995).

[5] P. H. Souto Ribeiro, S. Padua, J. C. Machado da Silva, and G. A. Barbosa, Phys. Rev. A 49, 4176 (1994).

[6] P. H. Souto Ribeiro, S. Padua, and C. H. Monken, Phys. Rev. A 60, 5074 (1999).

[7] B. E. A. Saleh, A. F. Abouraddy, A. V. Sergienko, and M. C. Teich, Phys. Rev. A 62, 043816 (2000). 
[8] A. F. Abouraddy, B. E. A. Saleh, A. V. Sergienko, and M. C. Teich, Phys. Rev. Lett. 87, 123602 (2001).

[9] A. F. Abouraddy, B. E. A. Saleh, A. V. Sergienko, and M. C. Teich, J. Opt. Soc. Am. B 19, 1174 (2002).

[10] A. F. Abouraddy, P. R. Stone, A. V. Sergienko, B. E. A. Saleh, and M. C. Teich, quant-ph/0311147.

[11] A. Gatti, E. Brambilla, and L. A. Lugiato, Phys. Rev. Lett. 90, 133603 (2003).

[12] A. Gatti, E. Brambilla, M. Bache, and L. A. Lugiato, Phys. Rev. A 70, 013802 (2004).

[13] A. Gatti, E. Brambilla, and L. A. Lugiato, Proc. SPIE 5161, 192 (2004).

[14] R. S. Bennink, S. J. Bentley, and R. W. Boyd, Phys. Rev. Lett. 89, 113601 (2002).

[15] R. S. Bennink, S. J. Bentley, R. W. Boyd, and J. C. Howell, Phys. Rev. Lett. 92, 033601 (2004).

[16] A. Gatti, E. Brambilla, L. A. Lugiato, and M. I. Kolobov, Phys. Rev. Lett. 83 (9), 1763 (1999).

[17] E. Brambilla, A. Gatti, L. A. Lugiato, and M. I. Kolobov, Eur. Phys. J. D 15, 127 (2001).

[18] P. Navez, E. Brambilla, A. Gatti, and L. A. Lugiato, Phys. Rev. A 65, 013813 (2002).

[19] A. Gatti, R. Zambrini, M. San Miguel, and L. A. Lugiato, Phys. Rev. A 68, 053807 (2003).

[20] E. Brambilla, A. Gatti, M. Bache, and L. A. Lugiato, Phys.
Rev. A 69, 023802 (2004).

[21] Z. Y. Ou, S. F. Pereira, H. J. Kimble, and K. C. Peng, Phys. Rev. Lett. 68, 3663 (1992).

[22] A. Kuzmich, I. A. Walmsley, and L. Mandel, Phys. Rev. Lett. 85, 1349 (2000).

[23] R. E. Slusher, P. Grangier, A. LaPorta, B. Yurke, and M. J. Potasek, Phys. Rev. Lett. 59, 2566 (1987).

[24] B. Yurke, P. Grangier, R. E. Slusher, and M. J. Potasek, Phys. Rev. A 35, 3586 (1987).

[25] C. Kim and P. Kumar, Phys. Rev. Lett. 73, 1605 (1994).

[26] F. Grosshans and P. Grangier, Eur. Phys. J. D 14, 119 (2001).

[27] J. Cheng and S. Han, Phys. Rev. Lett. 92, 093903 (2004).

[28] Y. Jiang, O. Jedrkiewicz, S. Minardi, P. Di Trapani, A. Mosset, E. Lantz, and F. Devaux, Eur. Phys. J. D 22, 521 (2003).

[29] O. Jedrkiewicz, Y. Jiang, P. Di Trapani, E. Brambilla, A. Gatti, M. Bache, and L. Lugiato, EH5-4-THU, European Quantum Electronics Conference, Munich, Germany, 2003 (unpublished).

[30] V. Dmitriev, G. Gurzadyan, and D. Nikogosyan, Handbook of Nonlinear Optical Crystals, Springer Series in Optical Sciences No. 64 (Springer, Berlin, 1999).

[31] R. Hanbury-Brown and R. Q. Twiss, Nature (London) 177, 27 (1956).

[32] M. Bache, E. Brambilla, A. Gatti, and L. Lugiato (unpublished). 